\title{
Quantal Response and Nonequilibrium Beliefs Explain Overbidding in Maximum-Value Auctions*
}

\author{
Colin Camerer $^{\dagger} \quad$ Salvatore Nunnari ${ }^{\ddagger} \quad$ Thomas R. Palfrey ${ }^{\dagger}$
}

May 20, 2016

\begin{abstract}
We report new experimental data on a simple common value auction to investigate the extent to which bidding can be explained by logit QRE, in combination with different models about bidder beliefs: cursed equilibrium, level-k, and cognitive hierarchy. There is a close correspondence between the predicted bidding patterns in those models and the distribution of observed bids. The pattern of median bids in the data consists of a combination of overbidding for with signals, and near-value-bidding with higher signals. Logit QRE with heterogeneous bidders approximates this pattern. Combining QRE with any of the other models of belief formation leads to a closer match with the data. All the estimated models predict only small treatment effects across different versions of the game, consistent with the data. We also reanalyze an earlier dataset for the same game (Ivanov, Levin and Niederle 2010), which exhibited much more overbidding, and reach similar qualitative conclusions.
\end{abstract}

*We are grateful for comments from seminar audiences at the 2010 North-American ESA Meeting in Tucson, the Security Market Auctions and IPOs Conference at Northwestern University, the University of Southern California, and Stanford University. We thank Asen Ivanov, Dan Levin, and Muriel Niederle for sharing their data and for detailed comments on an earlier draft. We are also grateful for helpful comments and suggestions from the referees and editor. Palfrey gratefully acknowledges the financial support of the National Science Foundation (SES-1426560), the Gordon and Betty Moore Foundation (SES-1158), and the Russell Sage Foundation.

${ }^{\dagger}$ Division of the Humanities and Social Sciences, California Institute of Technology, Pasadena, CA 91125.

${ }_{\ddagger}^{\ddagger}$ Department of Economics, Bocconi University, Milan, Italy 20136. 


\section{Introduction}

In some markets, agents assign a common value to an object, which is equal to a particular statistic of their privately observed value signals. Bidding in such markets often leads to a "winner's curse", in which the winning bid is systematically greater than the common value.

Two recent studies of common-value auctions include an interesting and strategically simple case in which the common value is the maximum of the signals of two agents (Bulow and Klemperer, 2002). Carrillo and Palfrey (CP 2011) study this economic environment in the context of two-person bilateral trade. Ivanov, Levin and Niederle (ILN 2010) study a twoperson second-price auction mechanism in which the common value is the maximum signal; we call this the maximum-value auction. In $\mathrm{CP}$, the equilibrium prediction is that there should be no trade, regardless of which statistic determines the common value (including the maximum). In ILN, if the common value is the maximum signal, the unique symmetric equilibrium is to bid one's signal.

Both studies actually report large and significant deviations from the Nash equilibrium. That is, trade occurs frequently in the CP experiment, and bids in the ILN experiment are often much higher than the bidders' signals (in fact, a large fraction of bidders bid more than 10 times the maximum object value at least once). $\mathrm{CP}$ conjecture that the deviations they observed can plausibly be the result of bounded rationality of the form captured by cursed equilibrium (CE; Eyster and Rabin, 2005) or analogy-based expectations (Jehiel 2005). ILN present evidence that they interpret as discrediting explanations based on models of limited strategic sophistication, including both CE and level-k models (LK; Stahl 1996, Crawford and Irriberri 2007a). The latter conclusion is surprising, because LK models and the closely related cognitive hierarchy model (CH; Camerer, Ho, and Chong 2004) account for many features of observed behavior in a wide variety of other experimental games, including standard common value auctions (Crawford, Costa-Gomes and Iriberri 2013), as well as field data (e.g., Brown, Lovallo, Camerer 2012 and citations therein). Costa-Gomes and Shimoji (CGS 2015) critique the ILN conclusion in a different way than we do, and argue that some of the results are not inconsistent with belief-based models. See further discussion of this in Section 4.

Using the maximum-value auction as our starting point, this paper takes a dual approach to look deeper into that conclusion. First, we conduct a new set of laboratory auctions with the same rules and procedures of the primary treatments explored by ILN. This allows us to 
identify whether the surprising findings are the result of an unusual set of data. Second, we adopt a structural estimation approach to evaluate the models of belief formation based on limited strategic sophistication and/or cursed beliefs. To provide an error structure for doing this, we combine these belief based models with quantal response equilibrium (QRE; McKelvey and Palfrey 1995). The main finding is that the observed patterns in bidding behavior are reasonably well accounted for by a quantal response equilibrium model of heterogeneous bidders, and this conclusion is even stronger with respect to the models that also include parameters for biased belief models of CE, LK, or CH. Thus, while perfect best response models fail to explain the observed bidding behavior in maximum value auctions, such a negative conclusion does not apply to models that incorporate payoff-responsive stochastic choice.

The intuition for why these models do so well in this particular game is rather simple. While it is a fact that there is a unique symmetric Bayesian-Nash equilibrium in which bidders bid their signals, the equilibrium is weak; indeed, it is just about as weak as an equilibrium can possibly be. If the other bidder is bidding according to the Nash equilibrium - that is, bid equal to signal - then every bid greater than or equal to your signal is a (weak) best response to Nash equilibrium. ${ }^{1}$ QRE imposes the assumption that beliefs about the distribution of other players' choices are accurate, but allows for imperfect (noisy) response. In maximumvalue auctions, a natural conjecture is that logit equilibria will typically entail a substantial frequency of overbidding, even for rather small levels of noise. This is because bidding above one's signal is a small mistake (in fact, not a mistake at all in the Nash equilibrium). But then, if in equilibrium many bidders are overbidding substantially, underbidding is nearly a best response because the probability of winning when bidding one's signal is very low. The CE effects will further increase the amount of overbidding, as is also possible when beliefs are formed according to quantal response versions of $\mathrm{LK}$ or $\mathrm{CH}$.

Following ILN, in our experiment subjects first participated in one treatment, which was followed immediately by a second surprise treatment. In the first treatment, Phase $I$, the signal distributions are uniform discrete distributions over the 11 integers $0-10$, and are independent for the two bidders (randomly) paired together in each round. In Phase I, subjects received each of the 11 integer signals exactly once - sampling without replacementin a sequence of 11 rounds. Subjects could bid any non-negative amount up to $\$ 1,000,000$. In the second treatment, called Phase II, subjects bid against the distribution of their own

\footnotetext{
${ }^{1}$ Even underbidding can be optimal in the asymmetric Nash equilibria of the game.
} 
previous signal-bid pairs sampled randomly from the Phase I auction against other people. It is crucial to note that, to disable learning, there is no feedback throughout the entire sequence of 22 bids (=11 bids per phase $\times 2$ phases).

Figure 1 shows scatter plots of actual bids and private signals in Phase I (panel a) and Phase II (panel b) of our experiments, excluding bids above $\$ 15$. There are two striking findings that are common to our data and ILN's. First, there is substantial overbidding which has a "hockey stick" shape: median bids when signals are below 5 are relatively flat, around $3-5$, only weakly responding to increases in the signal; bids when signals are above 5 are slightly above the signals, and steadily increase with signals. As we see below, this pattern arises very naturally from QRE. Second, median bids do not change very much between the Phase I two-player auction and the Phase II (bid-against-a-computerized-version-of yourown-phase-I) treatment. We show that these two empirical results are consistent with both quantal response equilibrium (QRE) and with quantal response versions of theories in which strategic thinking is limited by either cursed beliefs or levels of strategic sophistication.

Figure 1: Bids as a function of private signal values in our experiment: Phase I (a), and Phase II (b). Bids above 15 are not shown. The continuous line is the median bid as a function of private signal (considering all bids).

(a)

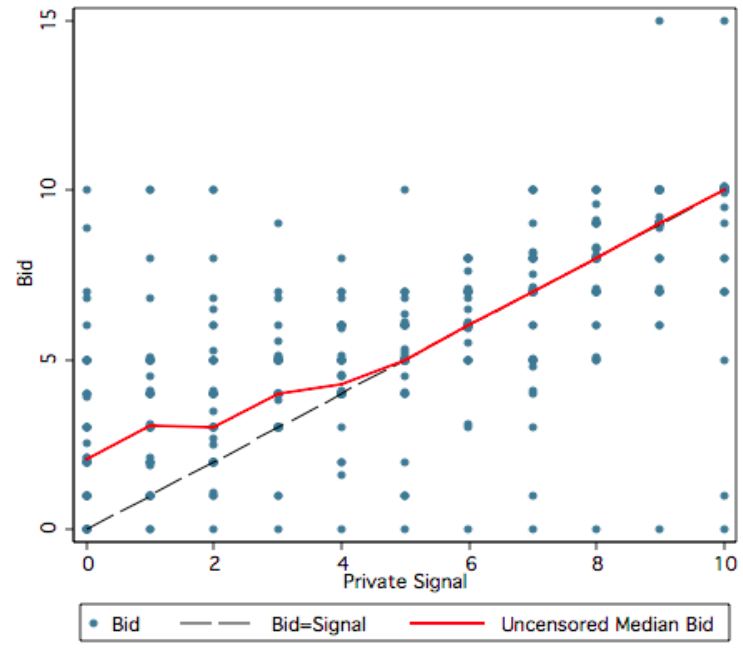

(b)

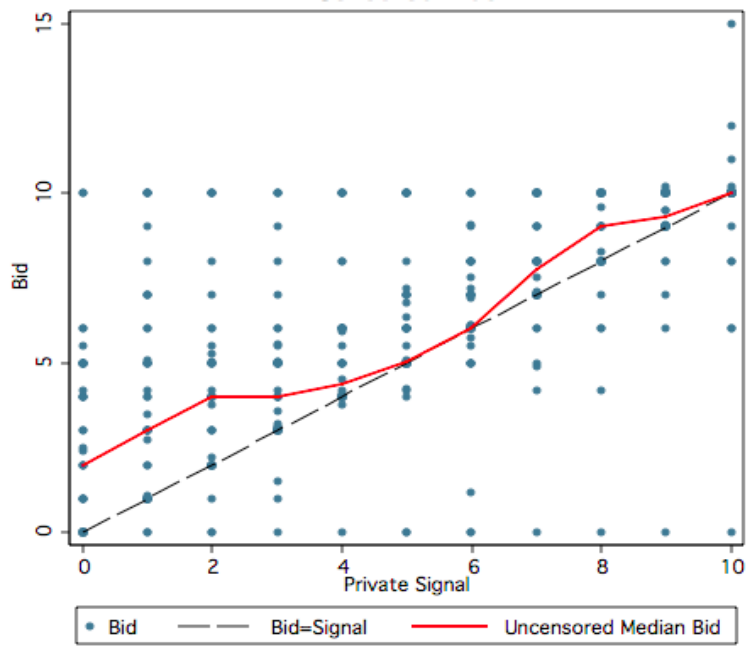

Note that accurate fits from QRE do not arise because of a loose intuition that 'quantal response can fit anything'. First, this assertion is simply not generally true for regular quantal 
response equilibria, including the most common logit and power specifications (Goeree, Holt and Palfrey, 2005). These equilibria satisfy various natural properties of continuity and responsiveness of choice probabilities to payoffs - higher expected payoffs lead to higher choice probabilities. If subjects' choices do not obey the latter property then regular QRE cannot fit the data well.

Second, our estimation procedure is designed to guard against overfitting the data. Specifically, the Phase I data are first used to derive QRE parameters, which fit those data best using maximum likelihood estimation. Then those estimates from Phase I are used to provide out-of-sample forecasts of Phase II behavior. In principle, these out-of-sample forecasts for average bidding behavior should be fairly close to the Phase I average bids, because of the relatively small treatment effects in both our data and the ILN data. This is what we find.

\subsection{The Winner's Curse}

The winner's curse results when bidders bid too high a fraction of a private value estimate, in common-value auctions, leading them to overpay (relative to equilibrium bidding) and even lose money. The winner's curse is one of the most interesting and best-documented facts in empirical auction analysis (e.g., Kagel and Levin 2002). The fundamental explanation for the observed overbidding has, surprisingly, proved to be elusive.

A likely cause contributing to overbidding is the failure of agents to appreciate the relation between the bids of other agents and those agents' perceived value of the auctioned good. This mistake is the motivation for $\mathrm{CE}$ and the related ABE model. Another possible cause is that agents simply find it conceptually difficult to compute a conditional expectation of value of any sort. If the difficulty of this computation is the main source of the winner's curse, the same mistake should also occur in a single-person decision where there is no strategic uncertainty, but there is identical conditional expectation. Indeed, there is some experimental evidence of such a mistake in decisions (e.g., Carroll and Bazerman 1988, Charness and Levin 2009). This fact is especially relevant to the experiment at hand, because it implies that even when bidders are explicitly told the joint distribution of bids and signals of the opponent (as in Phase II bidding), they still will not fully take account of the correlation between their opponent's bids and action.

What about strategic models of the winner's curse? One approach is CE. Another class of models includes the LK and $\mathrm{CH}$ specifications of beliefs which assume that individuals have 
different beliefs that correspond to different levels of strategic sophistication. Both specifications start with some clearly specified level-0 (L0) play. L0 choices are typically assumed to be random and uniform, but could more generally be considered instinctive (Rubinstein, 2007), focal, based on salience (Crawford and Iriberri, 2007a, 2007b), or corresponding to other types (Stahl and Wilson 1995).

Level-1 (L1) players assume they are playing L0 players. An iterated hierarchy then follows, most typically in two different ways: in "level-k" models Lk players (for $k \geq 1$ ) believe their opponents are all using Lk-1 reasoning. In $\mathrm{CH}$ models Lk players believe opponents are using a mixture of level $0,1, \ldots, k-1$ reasoning; their normalized beliefs, therefore, approach equilibrium beliefs as $\mathrm{k}$ increases.

The simplest forms of these models assume players choose exact best responses given their beliefs. However, the earliest forms of level-k and $\mathrm{CH}$ models typically replace this best response assumption with some kind of quantal response (Stahl and Wilson 1995; Ho, Camerer and Weigelt 1998; Costa-Gomes, Crawford and Broseta 2001, Chong, Ho and Camerer 2005). Indeed, models combining quantal response with $\mathrm{LK}$ or $\mathrm{CH}$ account for $60 \%$ of papers (37 out of 61 ) using level-k or $\mathrm{CH}^{2}$

This observation raises two interesting empirical questions: First, can quantal response combined with CE, LK or CH beliefs (denoted CE-QRE, LK-QRE, or CH-QRE) or equilibrium beliefs (QRE) explain the bids in maximum-value experiments? And, second, can such models explain why bids do not change much in the Phase II bid-against-yourself treatment? We answer these questions by estimating those models on Phase I data, seeing how well they fit, and then testing how well they fit Phase II bids (out of sample).

\subsection{The Maximum Value Game}

In the maximum-value second price auction, two players observe private signals $x_{1}$ and $x_{2}$ drawn independently from a commonly known distribution over the real line. They bid for an object which has a common value equal to the maximum of those two signals, $\max \left(x_{1}, x_{2}\right)$. The highest bidder wins the auction and pays a price equal to the second-highest bid. How should you bid? Intuitively, if your own value is high you might underbid to save some money. And if your own value is low you might overbid because the object value, determined by the maximum of the two values, is likely to be higher than your low value.

\footnotetext{
${ }^{2}$ See Camerer, Nunnari, and Palfrey 2012.
} 
These intuitions are not consistent with equilibrium, however. Bidding less than your signal is a mistake because your bid just determines whether you win, not what you pay, so bidding less never saves money. In fact, you might miss a chance to win at a price below its value if you bid too low and get outbid, so underbidding is weakly dominated. Second, bidding above your signal could be a mistake because if the other bidder is also overbidding, either of you may get stuck overpaying for an item with a low maximum-value. In the unique symmetric Bayesian-Nash equilibrium, therefore, players simply bid their values. In fact, the symmetric equilibrium where both players bid their signal can be solved for with two rounds of elimination of weakly dominated strategies, as shown in ILN.

While these bidding intuitions are mistakes, they can be small mistakes in terms of expected foregone payoffs. Moreover, payoff functions have many completely flat regions, so it turns out that some 'mistakes' of overbidding actually have zero expected cost in equilibrium (and hence are not necessarily mistakes). Whether mistakes are small or large is of central importance in the QRE approach. A key insight of quantal response equilibrium analysis is that even if large mistakes are rare, small mistakes could be common, and their equilibrium effects must be carefully analyzed. These equilibrium effects could be large, so small mistakes in payoff space can lead to large changes in the distribution of strategies. Therefore, even if over- and underbidding in maximum-value games are mistakes, those mistakes in bidding patterns could be common and could also have a substantial influence on bidding in general. Our paper explores the extent to which this possibility can explain the empirical results from laboratory experiments.

\subsection{How Predictions Change with Quantal Response Combined with Belief Based Models}

This section explains the three key ideas about the incentives to deviate from equilibrium behavior. First, we explain some intuitions of how bidding in Phase I depends on the change from an assumption of perfect best response behavior to quantal response behavior. Second, we explain the intuition for the effect of imperfect beliefs, à la CE and $\mathrm{LK}^{3}$ models, when combined with quantal response behavior. Third, we provide a theoretical intuition for why the difference in bidding behavior between Phase I and Phase II can be negligible under both

\footnotetext{
${ }^{3}$ For most of the analysis of models of strategic sophistication that follows we focus on LK rather than $\mathrm{CH}$, because it is easier to explain. The implications of those two models in this auction game are similar. We fit the data using both models and obtain nearly identical results.
} 
the quantal response models and the imperfect belief models.

First, to convey a sense of how QRE can approximate the behavior in Phase I, it is useful to consider optimal and nearly optimal responses to the bidding behavior of your opponent, under different hypotheses about his or her degree of quantal response behavior. Recall that in the logit version of quantal response, for each possible signal $x$, a bidder uses a behavioral strategy where the log probability of choosing each available bid is proportional to its expected payoff, where the proportionality factor, $\lambda$, can be interpreted as a responsiveness (or rationality) parameter. In particular, we have, for each $b \in B$ and for each signal $s$ :

$$
\operatorname{Pr}(b \mid s)=\frac{\exp (\lambda U(b \mid s))}{\sum_{a \in B} \exp (\lambda U(a \mid s))}
$$

where $B$ is the set of available bids, and $U(b \mid s)$ denotes the expected utility of bidding $b$ conditional on privately observing signal $s$. The key question then is how a player forms his expectations, in particular, how a bidder's beliefs about the other bidder affects the expected utility term, $U(b \mid s)$. QRE imposes rational expectations, or correct equilibrium beliefs, so $U(b \mid s)$ is computed using the QRE bidding strategies for a given value of $\lambda$.

At one extreme, if $\lambda$ equals infinity, then everyone believes that everyone is bidding perfectly, in the sense of bidding according to the unique symmetric Nash equilibrium. Thus beliefs boil down to believing that one's opponent will always bid his signal. However, if this is the case, then overbidding any amount is also a perfect best response, because if you win you will never pay more than your opponent's signal, which is less than or equal to the common value! Thus, in this case, which corresponds to behavior in a quantal response equilibrium with very high values of $\lambda$, we have $U(b \mid s) \approx U\left(b^{\prime} \mid s\right)$ for all $s \in\{0,1, \ldots, 10\}$ and for all $b, b^{\prime} \geq x$. Thus, in a quantal response equilibrium, for high levels of rationality, payoff functions are completely flat on the entire bidding space bounded below by $b=s$. In contrast, there are significant losses from underbidding if one's opponent is bidding his signal. This asymmetry of payoff losses has important consequences for QRE bidding strategies.

Next, consider the opposite extreme, $\lambda$ equal to 0 , where everyone believes everyone is bidding completely randomly. If this is the case, then there is no correlation between your opponent's signal and his bid, so there is in fact no possibility of a winner's curse. The expected value of the object to you is simply 
Figure 2: QRE strategies for different $\lambda$ 's, Phase I
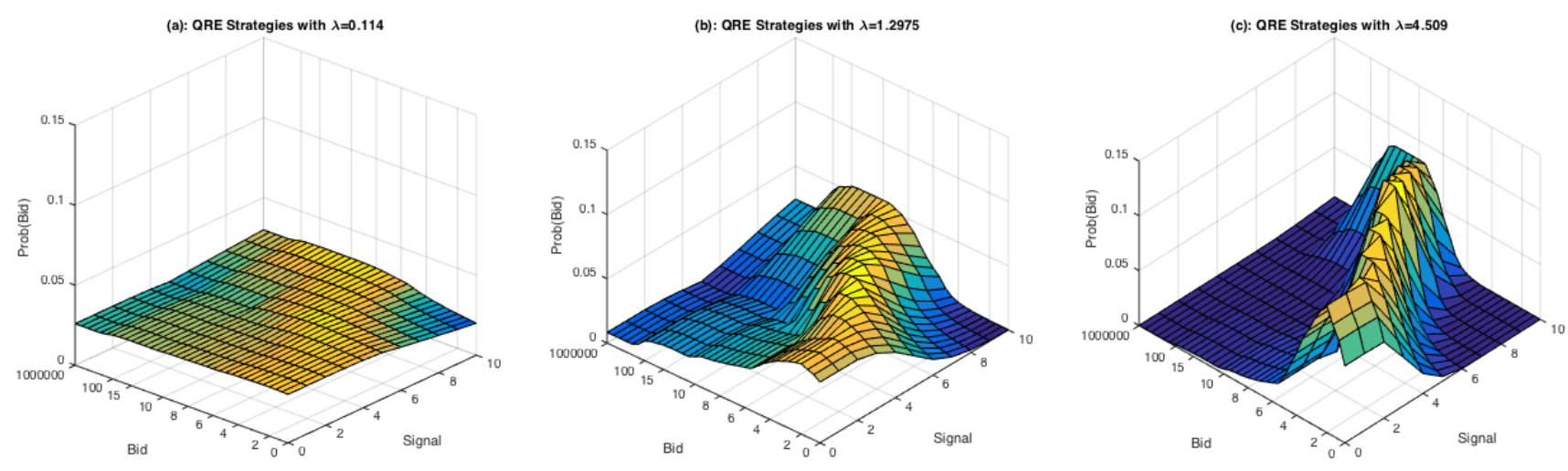

$$
E\left[\max \left\{s_{i}, s_{-i}\right\} \mid s_{i}\right]=\frac{s_{i}\left(s_{i}+1\right)}{11}+\frac{\left(10-s_{i}\right)\left(11+s_{i}\right)}{22} .
$$

for all bids $b \in B$ and for each signal $s$.

Straightforward calculations show that your optimal bid when $s_{i}=0$ equals 5 and the optimal bid increases monotonically to 10 when your value is 10 . For all values of $s_{i}$ except 10 , the optimal bid $\left(E\left[\max \left\{s_{i}, s_{-i}\right\} \mid s_{i}\right]\right)$ is strictly greater than $s_{i}$. Hence, the direct effect of quantal response behavior involves a combination of over-and underbidding, while the equilibrium effect of quantal responses pushes in the direction of overbidding.

Figure 2 shows three histograms of the theoretical joint distribution of bids and signals in the logit QRE, for three values of $\lambda(0.114,1.2975,4.509)$. The distribution begins rather flat for low values of $\lambda$ (left panel). As $\lambda$ increases (middle and right panel) the distribution of bids becomes more concentrated, with substantial overbidding for all signals. When there is a lot of random bidding (from players with low $\lambda$ values), it pays for low-signal bidders with higher $\lambda$ 's to overbid, since they have a chance to get a bargain on high-value items if the high-signal players underbid. Similarly, high-signal players with higher $\lambda$ 's bid their signals or higher because underbidding is a substantial mistake in expected payoff terms. The highest value of $\lambda$ gives a piecewise linear distribution that resembles an hockey-stick.

Of course, the discussion above simplifies the intuition of quantal response equilibrium; the exact joint distribution of bids and signals in a quantal response equilibrium depends in a complicated way on how all these equilibrium effects balance out. In fact, these interaction effects are even more complicated in the estimation of the next section, where we allow 
different bidders to have different response parameters, as in the heterogeneous version of QRE (HQRE) developed in Rogers, Palfrey, and Camerer (2009).

The effects of CE and LK complement, and in some cases reinforce, the effects of quantal response equilibrium. With respect to $\mathrm{CE}$, a stark way to see this is to note that if $\lambda=0$ there is no winner's curse, so quantal response equilibrium, for any positive value of $\lambda$ will necessarily embody some degree of partially cursed behavior. Both effects (CE and QRE) push bids up for low signal values, and reduce the expected losses from overbidding. In LK models, L1 bidders behave exactly as if they are facing $\lambda=0$, so the discussion of optimal responses is the same as described above. However, L2 bidders who think they are playing L1 know they are playing over-bidders so an optimal response is to bid their signal. In fact L2 bidders generally have a range of optimal bids conditional on signal. To consider even higher levels, recall that if an opponent is bidding their signal or lower, then overbidding and bidding one's signal are both weak best-responses. Therefore, an L3 bidder is actually expected to overbid against an L2 bidder. Following a similar logic, higher level players alternate from level to level between over and underbidding (or value bidding) with a range of optimal bids for each signal (k even) and (approximately) signal bidding except for the lowest signals ( $\mathrm{k}$ odd). Table 1 gives the range of optimal bids for levels, $k=1,2,3,4$. Notice that all four level types may overbid with low signals, and levels 0,2 , and 4 may overbid or underbid, even with the same signal, and million dollar bids are best responses for even types for some signal realizations. ${ }^{4}$

Thus, the LK model assuming best response, predicts a mixture of random bidding, overbidding, and signal-bidding, as in QRE. ${ }^{5}$

What are the implications of these models of quantal response and imperfect beliefs for Phase II bidding behavior? In Phase II, each bidder competes against a computerized player that is programmed to bid exactly according to the bidding function the bidder used in Phase I. Because there is no feedback about payoffs and auction outcomes between phases, in our analysis we assume that the degree of payoff responsiveness or cursedness is the same in Phase I and Phase II for each bidder, but each bidder still takes fully into account that their opponent's (stochastic) bidding strategy is governed by a different distribution in Phase I and Phase II.

\footnotetext{
${ }^{4}$ For $\mathrm{k}$ sufficiently large, odd types are value bidders and million dollar bids are best responses for even types for all signal realizations.

${ }^{5}$ Optimal bids for L2 and greater in the CH model are somewhat different from the LK bids shown in the table, and depend on the relative frequencies of lower levels.
} 
Table 1: Optimal Bids in Level-k Model

\begin{tabular}{ccccc} 
Signal & \multicolumn{4}{c}{ Level } \\
& 1 & 2 & 3 & 4 \\
0 & 5.00 & $0.00-4.99$ & 2.50 & $0.00-2.49$ \\
1 & 5.09 & $0.00-4.99$ & 2.67 & $0.00-2.49$ \\
2 & 5.27 & $0.00-4.99$ & 3.00 & $0.00-2.49$ \\
3 & 5.55 & $0.00-4.99$ & 3.50 & $2.68-3.49$ \\
4 & 5.91 & $0.00-4.99$ & 4.17 & $3.51-4.16$ \\
5 & 6.36 & $0.00-5.00$ & 5.00 & $5.00-10^{6}$ \\
6 & 6.91 & $5.92-6.35$ & 6.00 & $6.00-10^{6}$ \\
7 & 7.55 & $6.92-7.54$ & 7.00 & $7.00-10^{6}$ \\
8 & 8.27 & $7.56-8.26$ & 8.00 & $8.00-10^{6}$ \\
9 & 9.09 & $8.28-9.08$ & 9.00 & $9.00-10^{6}$ \\
10 & 10.00 & $9.10-10^{6}$ & 10.00 & $10.00-10^{6}$
\end{tabular}

First, consider the QRE model. In this case, if a bidder is a low- $\lambda$ bidder who is relatively unresponsive to payoff differences, we would expect a lot of variance in the form of under- and over-bidding in Phase II as well as Phase I. Since the bidders who grossly overbid in Phase I reveal themselves to have very low $\lambda$, this behavior is expected to persist in Phase II-indeed, that is also reflected in the data. On the other hand, bidders who bid close to their signals in Phase I will be identified as high $\lambda$ bidders and, in Phase II, they bid against bids close to signals (their own previous bids in Phase I). In this case, bidding signals and above signals have similar expected payoffs and imperfect best response actually implies these subjects may bid above their previous bids. Thus, QRE predicts rather small treatment effects across the two phases, with the possibility of an increase in bids between Phase I and Phase II, depending on the distribution of the responsiveness parameter.

What about the CE model in Phase II? While the players know the bidding strategy of the computerized opponent, they must bid without knowing the signal drawn by the opponent in a specific round. Thus, one can still apply the cursed equilibrium model, where one of the players (the computer) simply has a fixed strategy. And, as in CE, there is no reason to expect that a human bidder who fell prey to cursed beliefs in Phase I would have an epiphany between phases and suddenly realize how to correctly account for the correlation between the machine bidder's signals and bids. As noted in our introduction, behavior based on cursed beliefs has been widely observed in decision experiments with human players competing 
against computerized rules in situations that are otherwise isomorphic to games. Thus, in Phase II, as in Phase I, cursed behavior combined with QRE could produce a relatively modest increase in the extent of overbidding relative to a pure QRE model.

Finally, consider the implications of LK models for Phase II. With a perfect best response model, a level $\mathrm{k}$ bidder in Phase I should behave like a level $\mathrm{k}+1$ bidder in Phase II. For example, if a bidder is a value bidder (k odd) in Phase I, she should be an over-bidder in Phase II, and viceversa. This is not what one observes in the data. However, the picture changes in two interesting ways if one considers quantal response behavior instead of perfect best responses. First, bidders who are L0 or odd-level bidders in Phase I (because they overbid) could be revealing a low response sensitivity $\lambda_{i}$. For example, their (stochastic) bidding function could look like Figure $1 \mathrm{a}$ or $1 \mathrm{~b}$ above. If $\lambda_{i}$ is not too large, they might respond very little to knowing that they are bidding against their own previous Phase I bids. Second, bidders who bid close to their signals in Phase I will be identified as candidate evenlevel bidders. But when they are bidding against their own previous bids in Phase I (which are bids equal to signals), then bidding signals and bidding above signals are equally "best" responses. Even a little quantal response implies that these bidders may actually bid above their signals, and hence above their own previous bids.

So the combined effect of quantal response on the analysis of the response to the Phase II treatment is to dampen (depending on $\lambda$ ) the predicted effect of the level-1's bidding lower, and to add an effect of level-2's bidding higher. Thus, it is not clear there will be any strong effect overall in bidding behavior in the comparison of Phase I and Phase II. ${ }^{6}$

\section{Procedures and Data}

Signals are uniformly, independently, and discretely distributed from 0 to 10 in integer increments. The baseline auctions are called "Phase I" auctions, and strategies are elicited using a particular form of the "strategy method". 7 This is done by eliciting from each bidder one bid for each of the 11 possible signals in a random sequence. Bids could be any non-negative amount up to $\$ 1,000,000$ in penny increments. Payoffs are determined for each of these signal-bid pairs by random matching with an opponent, but there is no feedback until both

\footnotetext{
${ }^{6}$ The same conclusion holds for the $\mathrm{CH}$ model with quantal response, which predicts identical L1 behavior and a similar L2 response to the Phase II treatment.

${ }^{7}$ See, for example, Selten (1967) and Roth (1995, p. 322-323).
} 
phases of the experiment finish. Phase II auctions were conducted immediately following the Phase I auctions. In Phase II, each subject again submitted 11 bids, one for each possible signal. These bids were then randomly matched against a computer that was programmed to use exactly the same bidding function that had been used by the same subject in Phase I. Subjects were told this before preparing their Phase II bids.

Our experiment was conducted at the Social Sciences Experimental Laboratory (SSEL), using students from the California Institute of Technology. Subjects were recruited from a database of volunteer subjects. Three sessions were run, using a total of 46 subjects. No subject participated in more than one session and each session lasted around 45 minutes. Instructions for Phase I were read aloud while subjects read along. After that, subjects were required to correctly answer all questions on a short comprehension quiz before the experiment was conducted. After Phase I, the experimenter read the instructions for Phase II. After Phase II, subjects were paid. Payoffs were converted to U.S. dollars using an exchange rate of $\$ 0.50$ US per experimental dollar. Subjects were paid $\$ 10$ plus or minus the total of all the payoffs from all auctions. Average earnings were 25.42 dollars. As in most common value auction experiments, extreme overbidding can lead to bankruptcy, although no subject in our experiment had negative earnings the auctions. 8 The experiment was programmed and conducted with the software z-Tree (Fischbacher 2007). ${ }^{9}$

\section{Model Estimation and Forecasting}

The figures presented in the Introduction, while informative of the overall pattern of bidding, conceal the extensive heterogeneity in bidding behavior across subjects. To illustrate the degree of heterogeneity in a very compact graphical format, Figure 3 graphs the empirical inverse cumulative distribution function of (unconditional) average bids by subject for Phase I data. There is a significant degree of heterogeneity: $24 \%$ of subjects have an average bid smaller than $5 ; 35 \%$ between 5 and $6 ; 41 \%$ greater than $6 .^{10}$

Because of the importance of heterogeneity, we consider in this section three different

\footnotetext{
${ }^{8}$ While this was never the case in our experiment, if a subject had negative cumulative earnings at the end, she would have been paid only her show-up fee, as in ILN.

${ }^{9}$ Our procedures were identical to one of the ILN treatments, including using the same exact instructions and software. The instructions are included in the Appendix. In ILN, with the same procedures, average earnings were $\$ 18.03$ and $28 \%$ of the subjects lost money from the auctions.

${ }^{10}$ The average bid of a value bidder is 5 .
} 
Figure 3: CDF of Mean bids, Phase I

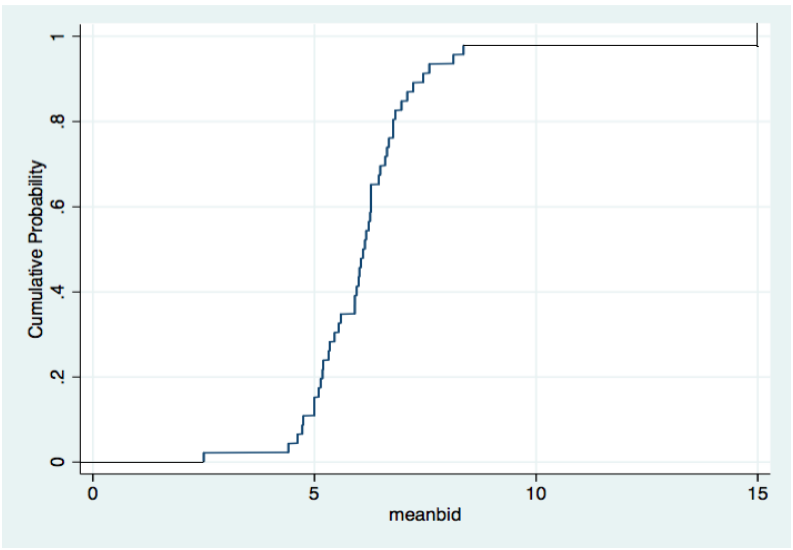

models of bidding behavior that incorporate different kinds of heterogeneity. The first kind of heterogeneity we consider is the degree to which subjects are best-responding, which we call skill heterogeneity. Specifically, individuals differ in their logit response parameter $\lambda$, with low values corresponding to low skill (or weak payoff responsiveness) and high values corresponding to high skill (or high payoff responsiveness). Significant evidence of such heterogeneity has been reported elsewhere (Rogers et al. 2009) and can be thought of as a reduced form approach to modeling differences in skill, carelessness, confusion, and other factors affecting ability of players to choose perfect best responses. For reasons discussed earlier, the relatively flat payoffs and the fact that best responses are generally not unique in this game suggest that this kind of heterogeneity may help explain some of the variation of bidding behavior across players.

The second kind of heterogeneity, captured by the LK and $\mathrm{CH}$ modeling approach, has also been discussed earlier and explicitly incorporates a model of heterogeneity with respect to beliefs; it is one of the primary motivations behind the experimental design. In our estimation, we assume that Lk players believe all their opponents are (quantal responding) Lk-1 players, and that the frequency of Lk players in the population follows a Poisson distribution. ${ }^{11}$

\footnotetext{
${ }^{11}$ We also estimated the $\mathrm{CH}$ version of the level-k model (Camerer, Ho, and Chong 2004) where step $k$ players believe that their opponents are distributed according to a normalized Poisson distribution, from step 0 to step $k-1$. Therefore, they accurately predict the relative frequencies of less sophisticated players, but ignore the possibility that some players may be as sophisticated or more. Step 1 players assume that all the other players are step 0 players, step 2 players assume the other players are a combination of step 0 players and (quantal responding) step 1 players, and so on. The estimates for this alternative model are very similar to the estimates for the regular version of level-k.
} 


\subsection{QRE and HQRE Models Estimation}

In the logit version of HQRE, all players have common rational expectations (and hence no belief heterogeneity) as in QRE, but can have different response parameters. That is, players exhibit skill heterogeneity. Thus each player uses a behavioral strategy where the log probability of choosing each available action is proportional to its (rational expectations) expected payoff, where the proportionality factor, $\lambda_{i}$ varies across subjects. In particular, for our setting, we have:

$$
\operatorname{Pr}(b \mid x)=\frac{\exp \left(\lambda_{i} U(b \mid s)\right)}{\sum_{a \in B} \exp \left(\lambda_{i} U(a \mid s)\right)}
$$

for all bids $b \in B$ and for each signal $s$.

In the maximal value auction, when agent $i$ receives private signal $s_{i}$, his expected utility from bid $b_{i}$ is the following:

$$
\begin{aligned}
E U\left(b_{i} \mid s_{i}\right)= & \operatorname{Pr}\left(b_{i}>b_{j}\right)\left[E\left(\max \left(s_{i}, s_{j}\right) \mid b_{i}>b_{j}\right)-E\left(b_{j} \mid b_{i}>b_{j}\right)\right] \\
& +\frac{1}{2} \operatorname{Pr}\left(b_{i}=b_{j}\right)\left[E\left(\max \left(s_{i}, s_{j}\right) \mid b_{i}=b_{j}\right)-b_{i}\right]
\end{aligned}
$$

The first term is the probability of winning times the expected net benefit conditional on winning, while the second term is the probability of a tie times the expected net benefit conditional on tying (ties are broken randomly). Given these expected payoffs, the choice probabilities follow from the choice rule in (2), i.e.:

$$
\operatorname{Pr}\left(b_{i} \mid s_{i}\right)=\frac{\exp \left(\lambda_{i} E U\left(b_{i} \mid s_{i}\right)\right)}{\sum_{a \in B} \exp \left(\lambda_{i} E U\left(a \mid s_{i}\right)\right)}
$$

An insight from Bajari and Hortacsu (2005) enables us to estimate HQRE consistently. They note that, if the model is correct, then the actual data provide an unbiased estimator of the aggregate joint distribution of bids and signals - and also players' beliefs, since beliefs are assumed to be consistent with actual behavior, by the 'E' part of the definition of QRE.

Therefore, we use the empirical joint distributions of signals and bids (i.e., the distributions observed in the experiments) in Phase I auctions to compute the empirical expected utility of each bid given each private signal, $\widehat{E U}\left(b_{i} \mid s_{i}\right){ }^{12}$ Specifically, we compute $\widehat{E U}\left(b_{i} \mid s_{i}\right)$

\footnotetext{
${ }^{12}$ For tractability, we bin bids for the estimation. The bins use $\$ .50$ intervals for bids less than or equal to
} 
for each (binned) bid $b_{i}$ and each signal $s_{i}$ in Phase I as follows:

- $\operatorname{Pr}\left(b_{i}>b_{j}\right)$ is the fraction of bids submitted by all subjects in Phase I lower than $b_{i}$;

- $E\left(\max \left(s_{i}, s_{j}\right) \mid b_{i}>b_{j}\right)$ is the average value of the object in all Phase I auctions where a subject bids less than $b_{i}$;

- $E\left(b_{j} \mid b_{i}>b_{j}\right)$ is the average bid among all bids strictly lower than $b_{i}$ submitted by all subjects in Phase I;

- $\operatorname{Pr}\left(b_{i}=b_{j}\right)$ is the fraction of bids submitted by all subjects in Phase I equal to $b_{i}$;

- $E\left(\max \left(s_{i}, s_{j}\right) \mid b_{i}=b_{j}\right)$ is the average value of the object in all Phase I auctions where a subject bids exactly $b_{i}$.

Then, for each subject $i$, we estimate, using MLE, the response parameter $\widehat{\lambda_{i}}$ that best fits $i$ 's observed bidding function. This gives us an estimated distribution over bids (conditional on the signal) for each subject. Aggregating over all subjects, we obtain a fitted distribution of bids for each signal in Phase I that can be compared to the empirical joint distribution of bids and signals.

Next, for Phase II, we compute out-of-sample predictions about bidding behavior based on the parameters estimated from Phase I. This is done in the following way. For each subject, we first compute $\widehat{E U}_{i}\left(b_{i} \mid s_{i}\right)$ using the empirical joint distribution of bids and signals from his previous play in Phase I (i.e., each agent plays against his exact previous bidding function). Specifically, we compute $\widehat{E U}_{i}\left(b_{i} \mid s_{i}\right)$ for each (binned) bid $b_{i}$, each signal $s_{i}$, and each subject $i$ in Phase II as follows:

- $\operatorname{Pr}\left(b_{i}>b_{j}\right)$ is the fraction of bids submitted by $i$ in Phase I lower than $b_{i}$.

- $E\left(\max \left(s_{i}, s_{j}\right) \mid b_{i}>b_{j}\right)$ is the average value of the object in all Phase I auctions where $i$ bids less than $b_{i}$;

- $E\left(b_{j} \mid b_{i}>b_{j}\right)$ is the average bid among all bids strictly lower than $b_{i}$ submitted by $i$ in Phase I;

- $\operatorname{Pr}\left(b_{i}=b_{j}\right)$ is the fraction of bids submitted by $i$ in Phase I equal to $b_{i}$. 
- $E\left(\max \left(s_{i}, s_{j}\right) \mid b_{i}=b_{j}\right)$ is the average value of the object in all Phase I auctions where $i$ bids exactly $b_{i}$.

Finally, we predict each subject's behavior in Phase II by plugging in these expected payoffs and the $\widehat{\lambda_{i}}$ estimated for Phase I into equation (3). ${ }^{13}$ This yields the out-of-sample (stochastic) prediction for player i's bids. Aggregating over all subjects, we obtain the outof-sample predicted distribution of bids for each signal in Phase II that we can then compare to the empirical joint distribution of bids and signals.

In addition to estimating the HQRE model with heterogenous $\lambda_{i}$ 's, we estimate an homogenous QRE model where we constraint all the $\lambda_{i}$ 's to be equal. Because the QRE model is nested in the HQRE model, this allows us to formally test for heterogeneity in the data using a likelihood ratio test. The results of the estimation for both models (QRE and HQRE) are summarized in Table 2 .

Figure 4 displays the median bids of the fitted distribution for Phase I and the forecast median bids for Phase II, and compares them with the quartiles of the observed bids (represented with box plots). The comparison has several notable properties. First, the estimated median bid functions generated are qualitatively very similar: both models capture the 'hockey-stick' pattern in the data, with relatively flat bidding for low signals and increasing bidding for high signals. Second, the heterogenous model fits the data much better than the homogeneous model (likelihood ratio greater than 260), with the homogeneous model clearly rejected at any conventional significance level (p-value of a Likelihood Ratio Test $<0.01$ ). Third, the Phase II out-of-sample predictions have a similar hockey-stick shape as the data, but the forecast median bids for higher values are greater than the observed median bids. That is, empirical bids in Phase II are somewhat lower than the model forecasts. Finally, it is important to note that the Phase II forecast bids are not lower than the Phase I forecast bids and this is also a feature of the observed bids; in fact, the distribution of bids is generally higher going from Phase I and Phase II. ${ }^{14}$

\footnotetext{
${ }^{13}$ Another common procedure is to fit models in each phase using part of the data, then predict for a holdout validation sample. We have not done this but conjecture that the results would be similar, except for the caveat that obvious heterogeneity in the data implies that hold-out forecasts for some outlying subjects will be poor.

${ }^{14}$ The reason for this is straightforward: in the data, many subjects bid their signal when they receive a high signal in Phase I; given this Phase I bidding behavior, their best response when they receive a high signal in Phase II can be any bid equal to their signal or higher. Hence, except for low signals, the median bid forecast for such bidders is higher in Phase II than in Phase I. This may also account for the slight increase in the distribution of observed Phase II bids relative to Phase I.
} 
Table 2: Estimated Parameters

\begin{tabular}{|c|c|}
\hline $\begin{array}{l}\text { \# Individuals } \\
\text { \# Observations }\end{array}$ & $\begin{array}{c}46 \\
506 \\
\end{array}$ \\
\hline \multicolumn{2}{|l|}{ QRE } \\
\hline$\widehat{\lambda}$ & 3.59 \\
\hline -Log-likelihood & 1447.8 \\
\hline \multicolumn{2}{|l|}{ HQRE } \\
\hline$\widehat{\lambda_{i}}$ Quartiles & $(3.40,5.12,8.70)$ \\
\hline -Log-likelihood & 1317.6 \\
\hline \multicolumn{2}{|l|}{ CE-QRE } \\
\hline$\widehat{x}$ & 0.1 \\
\hline$\widehat{\lambda}$ & 3.66 \\
\hline -Log-likelihood & 1440.4 \\
\hline \multicolumn{2}{|l|}{ CE-HQRE } \\
\hline$\widehat{\chi}$ & 0.2 \\
\hline$\widehat{\lambda_{i}}$ Quartiles & $(3.05,6.55,11.81)$ \\
\hline -Log-likelihood & 1289.7 \\
\hline \multicolumn{2}{|c|}{ LK-QRE (4 Types) } \\
\hline$\widehat{\tau}$ & 2.7 \\
\hline$\widehat{\lambda}$ & 12.93 \\
\hline Types Frequency & $(9 \%, 25 \%, 34 \%, 31 \%)$ \\
\hline -Log-likelihood P1 & 1346.3 \\
\hline \multicolumn{2}{|c|}{ LK-HQRE (4 Types) } \\
\hline$\widehat{\tau}$ & 4 \\
\hline$\widehat{\lambda_{i}}$ Quartiles & $(3.82,11.06,47.51)$ \\
\hline Types Frequency & $(4 \%, 17 \%, 34 \%, 45 \%)$ \\
\hline -Log-likelihood P1 & 1280.9 \\
\hline
\end{tabular}


Figure 4: HQRE (Top Panel) and QRE (Bottom Panel) Estimates
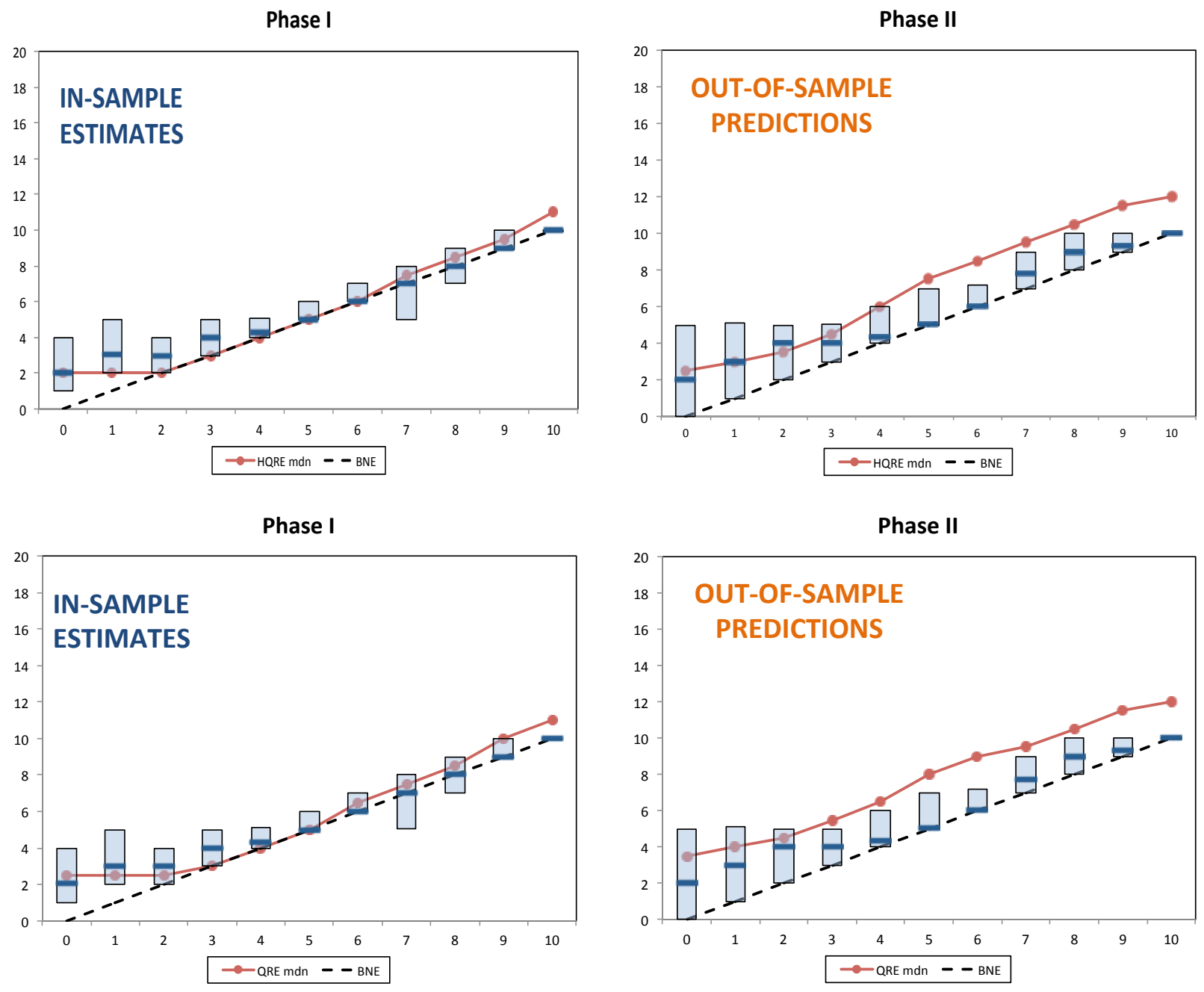

\subsection{CE-QRE and CE-HQRE Models Estimation}

An agent $i$ who is cursed, in the sense of Eyster and Rabin (2005), has correct expectations about the marginal distribution of other players' bids, but has incorrect beliefs about the joint distribution of bids and signals; specifically, a (fully) cursed bidder fails to account for the fact that bids are correlated with private signals. As a result, the expected utility of a 
cursed agent is given by:

$$
\begin{aligned}
E U_{C E}\left(b_{i} \mid s_{i}\right)= & \operatorname{Pr}\left(b_{i}>b_{j}\right)\left[E\left(\max \left(s_{i}, s_{j}\right)-E\left(b_{j} \mid b_{j}<b_{i}\right)\right]\right. \\
& +\frac{1}{2} \operatorname{Pr}\left(b_{i}=b_{j}\right)\left[E\left(\max \left(s_{i}, s_{j}\right)-b_{i}\right]\right.
\end{aligned}
$$

The extent to which agents are cursed is parameterized by the probability $\chi \in[0,1]$ they assign to other players playing their average distribution of actions irrespective of type rather than their actual type-contingent strategy (to which they assign probability $1-\chi$ ). In the maximal value game, the expected utility of a $\chi$-cursed agent $i$ from bidding $b_{i}$ when observing signal $s_{i}$ is given by:

$$
E U_{C E}^{\chi}\left(b_{i} \mid s_{i}\right)=(1-\chi) E U\left(b_{i} \mid s_{i}\right)+\chi E U_{C E}\left(b_{i} \mid s_{i}\right)
$$

A bid function, $\beta$, is a (symmetric) $\chi$-Cursed Equilibrium, if, for each bidder and for each $s, \beta(s)$ maximizes $E U_{C E}^{\chi}(b \mid s)$, given the other bidder uses $\beta$. The definition of $\chi$-Cursed HQRE ( $\chi$-CE HQRE) is given by the solution to the system of logit equations (3), replacing $E U(b \mid s)$ by $E U_{C E}^{\chi}(b \mid s)$ in the right hand side.

For estimation, as in Section 3.1, we use the empirical joint distributions of signals and bids in Phase I auctions to compute empirical values of $\widehat{E U}_{C E}^{\chi}\left(b_{i} \mid s_{i}\right)$. $\widehat{E U}\left(b_{i} \mid s_{i}\right)$ and most elements in $\widehat{E U}_{C E}\left(b_{i} \mid s_{i}\right)$ are computed as described in the previous section. One element in $E U_{C E}\left(b_{i} \mid s_{i}\right)$ is not part of $E U\left(b_{i} \mid s_{i}\right)$, that is, $E\left(\max \left(s_{i}, s_{j}\right)\right)$. This is the unconditional value of the object given your signal, that is, the value of the object assuming there is no correlation between the opponent's signal and his bid, as given by equation (1). Then, we jointly estimate with a standard MLE procedure the individual $\widehat{\lambda_{i}}$ 's and the cursed parameter $\hat{\chi}$ that best fits the data. We then compute the out-of-sample predictions for Phase II as in Section 3.1, and also estimate an homogenous model where the $\lambda_{i}$ 's are constrained to be the same.

Table 2 shows the results of the estimation, and Figure 5 shows the fitted distribution for Phase I and the predicted distribution for Phase II for both the CE-QRE and CE-HQRE models. The fitted distributions for Phase I are shown in the left-hand side of Figure 5. The out-of-sample predictions for Phase II are in the right-hand side of the same figure.

Intuitively, $\mathrm{CE}$ generates overbidding in Phase I because players ignore, to an extent calibrated by $\chi$, the connection between the bid and signal of the other player. Therefore, 
bidders with low signals do not realize that a low bid indicates the other player's signal may be low, and hence overbid to take advantage of the perceived bargain. The results are very similar to the QRE and HQRE estimations from the previous section. The CE-QRE and CE-HQRE models yield estimated and predicted median bidding functions with the same hockey-stick pattern as the median bids in the data. The predicted bids for Phase II slightly overshoot the median bids in the data, but less so than in the pure QRE models. And the CE model with heterogeneous $\lambda_{i}$ 's fits the data significantly better than the homogenous $\lambda$ model $(p<0.01)$. Both CE models fit the data significantly better than the pure QRE and HQRE models, suggesting that cursed beliefs may be a factor affecting bidding behavior. However, such an effect, if it exists, seems to play a relatively minor role. While the improvement in fit is statistically significant, the estimated degree of cursedness is low $(\widehat{\chi}=0.1$ for CE-QRE and $\widehat{\chi}=0.2$ for CE-HQRE), and the figures show that the estimated bidding functions are similar to the QRE and HQRE bidding functions, both qualitatively and quantitatively. The estimated distribution of $\widehat{\lambda_{i}}$ 's from the CE-HQRE model is similar to the one from the HQRE model as well, but with slightly more variation.

\subsection{LK-QRE and LK-HQRE Models Estimation}

For the LK estimation, denote the expected utility of step 1 players as $E U_{L K_{1}}\left(b_{i} \mid s_{i}, \tau\right)$, the one of step 2 players as $E U_{L K_{2}}\left(b_{i} \mid s_{i}, \tau\right)$, and the one of step 3 players as $E U_{L K_{3}}\left(b_{i} \mid s_{i}, \tau\right)$. We assume the presence of up to 4 levels of beliefs distributed according to a (truncated) Poisson frequency distribution $f(k)$ of step $k$ players in the population, with mean level equal to $\tau .{ }^{15}$ We assume that each agent is randomly drawn from this distribution and that he responds imperfectly as in an HQRE with an individual specific $\lambda_{i}$ :

$$
\begin{aligned}
\operatorname{Pr}\left(b_{i} \mid s_{i}\right)= & f(0) \frac{\exp \left(\lambda_{i} E U_{L K_{0}}\left(b_{i} \mid s_{i}\right)\right)}{\sum_{a \in B} \exp \left(\lambda_{i} E U_{L K_{0}}\left(a \mid s_{i}\right)\right)}+f(1) \frac{\exp \left(\lambda_{i} E U_{L K_{1}}\left(b_{i} \mid s_{i}\right)\right)}{\sum_{a \in B} \exp \left(\lambda_{i} E U_{L K_{1}}\left(a \mid s_{i}\right)\right)} \\
& +f(2) \frac{\exp \left(\lambda_{i} E U_{L K_{2}}\left(b_{i} \mid s_{i}\right)\right)}{\sum_{a \in B} \exp \left(\lambda_{i} E U_{L K_{2}}\left(a \mid s_{i}\right)\right)}+f(3) \frac{\exp \left(\lambda_{i} E U_{L K_{3}}\left(b_{i} \mid s_{i}\right)\right)}{\sum_{a \in B} \exp \left(\lambda_{i} E U_{L K_{3}}\left(a \mid s_{i}\right)\right)}
\end{aligned}
$$

${ }^{15}$ To keep the estimation simple, we only consider types $k \leq 3$. Allowing for a fifth level type in the estimation does not improve the fit. 
Figure 5: CE-HQRE (Top Panel) and CE-QRE (Botton Panel) Estimates
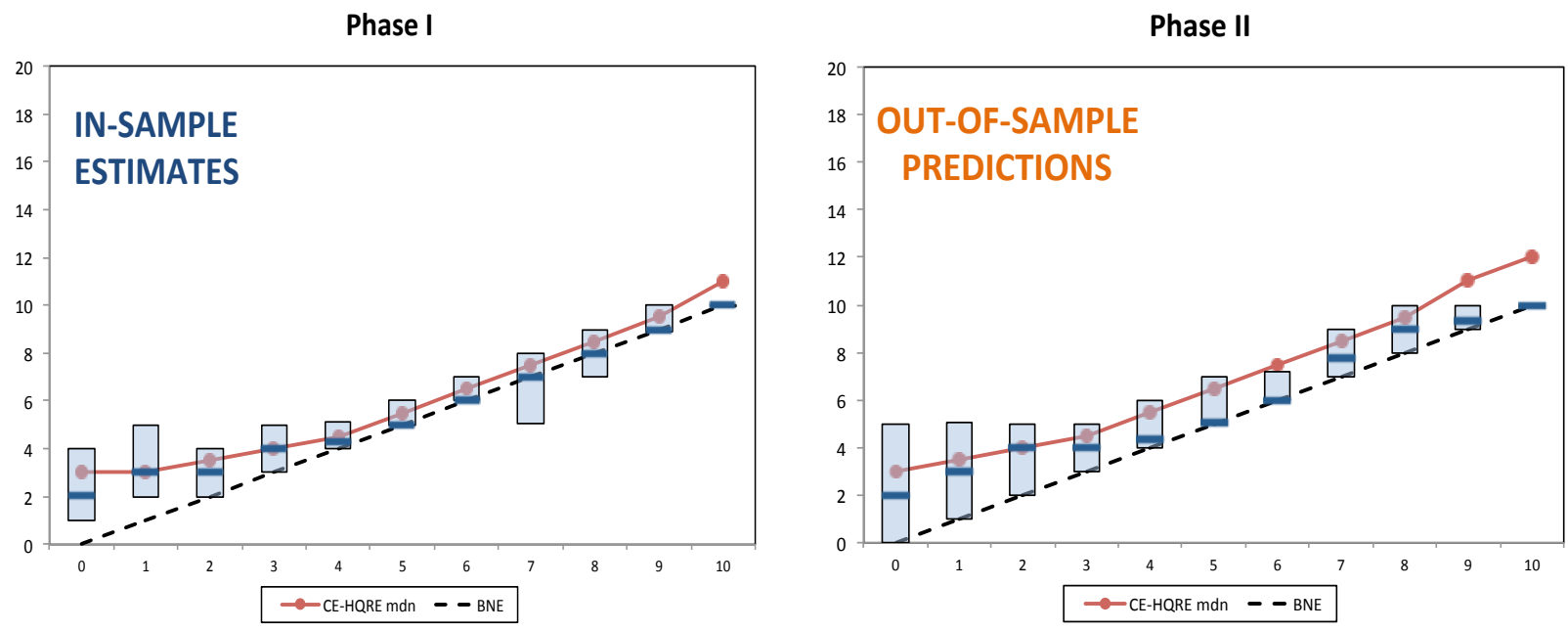

Phase I
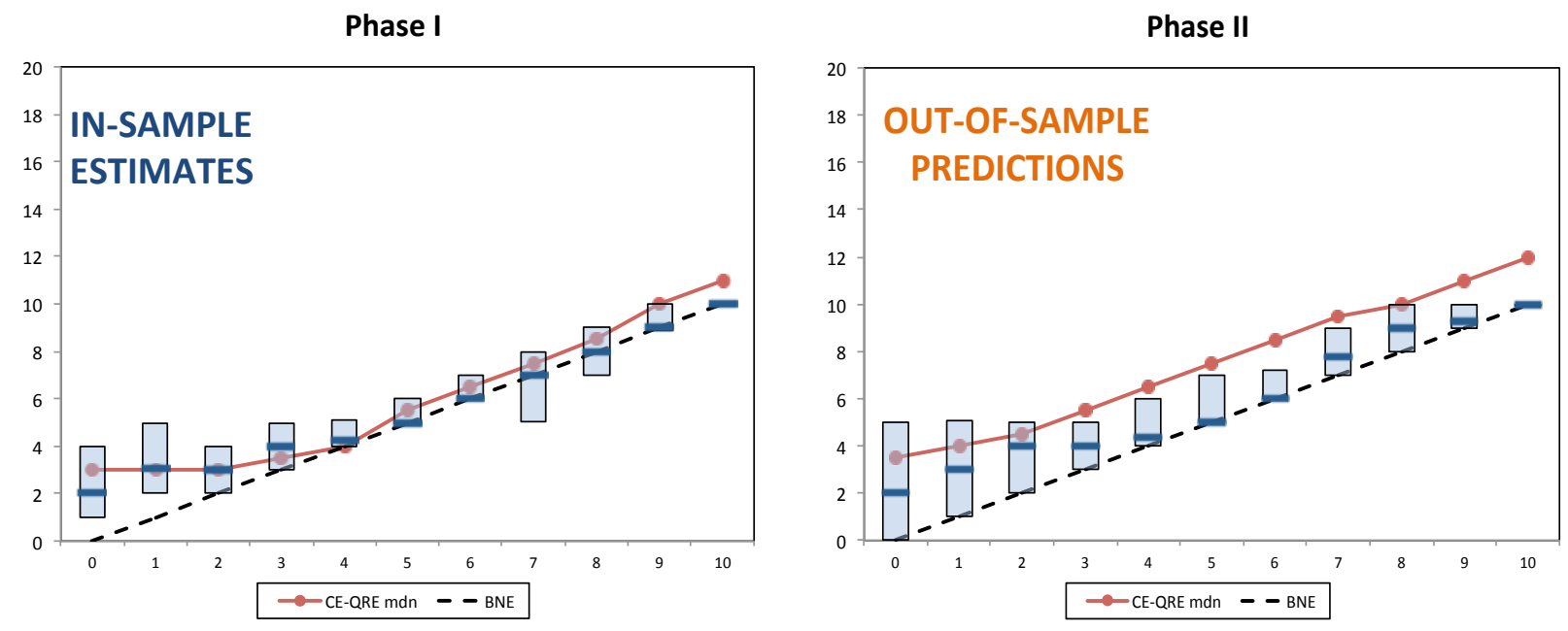

where $f(k)=e^{-\tau} \tau^{k} / k$ ! is the probability a subject belongs to level $k$.

For each pair $\left(\lambda_{i}, \tau\right)$, (5) implies a joint distribution of bids and signals for a subject. To generate the predictions for Phase I, we estimate with MLE the vector $\left(\widehat{\lambda}_{1}, \widehat{\lambda}_{2}, \ldots, \widehat{\lambda}_{N}, \widehat{\tau}\right)$ that fits best the empirical strategies from Phase I (where $N$ is the number of subjects in the experiment). Contrary to what we do for QRE and CE-QRE, we do not use the empirical joint distributions of signals and bids in Phase I auctions to compute the empirical expected utility of each bid given each private signal. Rather, we compute the expected utility of each bid given each private signal and the theoretical behavior of the lower level types. The 
expected utility of L1's is computed assuming they are facing L0's who choose any bid with the same probability, regardless of the signal. The expected utility of L2's (L3's) is computed assuming they are facing L1's (L2's) who imperfectly respond to L0's (imperfectly responding L1's).

For the out-of-sample predictions for Phase II, the estimated parameter for the Poisson distribution of types, $\widehat{\tau}$, has no role in Phase II, as players are now bidding against their own previous bid profile rather than beliefs that they are bidding against lower level players. ${ }^{16}$ In

addition to the fact that $\widehat{\lambda_{i}}$ is potentially different for every agent, we also take into account that each bidder has different beliefs (based on the empirical distribution of her previous bids in Phase I) and, thus, different expected payoffs.

Table 2 shows the results of the estimation and Figure 6 shows the fitted distribution for Phase I and the predicted distribution for Phase II for both the LK-HQRE and LK-QRE models. The estimates from the two models are qualitatively similar, and again the model with homogeneous $\lambda$ is soundly rejected by a likelihood ratio test $(p<0.01)$. The pattern of both estimated bids in Phase I and predicted Phase II bids track the data in a similar way as the other models. Among all models considered here, the LK-HQRE model, which combines skill heterogeneity (HQRE) with heterogeneity with respect to beliefs (LK) fits the data best.

\subsection{Summary and Interpretation of Results}

The similarities and differences between the estimation of these three classes of models (QRE/HQRE, CE-QRE/CE-HQRE, and LK-QRE/LK-HQRE) provide a number of insights about bidding behavior in these games, and how it relates to equilibrium. First, all three classes of models examined here track the basic pattern of bidding functions in the data. This is true both for the in-sample estimates from Phase I and the out-of-sample estimates from Phase II, although all models generate predictions that somewhat overshoot the empirical bid functions in Phase II, at least for a subset of signals. This is partly (or perhaps entirely) due to the fact that a number of bidders in our sample are almost perfect signal-bidders in Phase I. As pointed out earlier, all bids greater than or equal to your signal are optimal

\footnotetext{
${ }^{16}$ As CGS have pointed out, Phase II of these experiments are simple one person decision problems not interactive games, so level $\mathrm{K}$ theory does not apply. Thus the out-of-sample forecast bids for the LKHQRE model are not based on level-K theory at all, but depends only on the Phase I estimates of their individual logit response parameter under the LKHQRE model and their own Phase I bidding function.
} 
Figure 6: LK-HQRE (Top Panel) and LK-QRE (Bottom Panel) Model Estimates
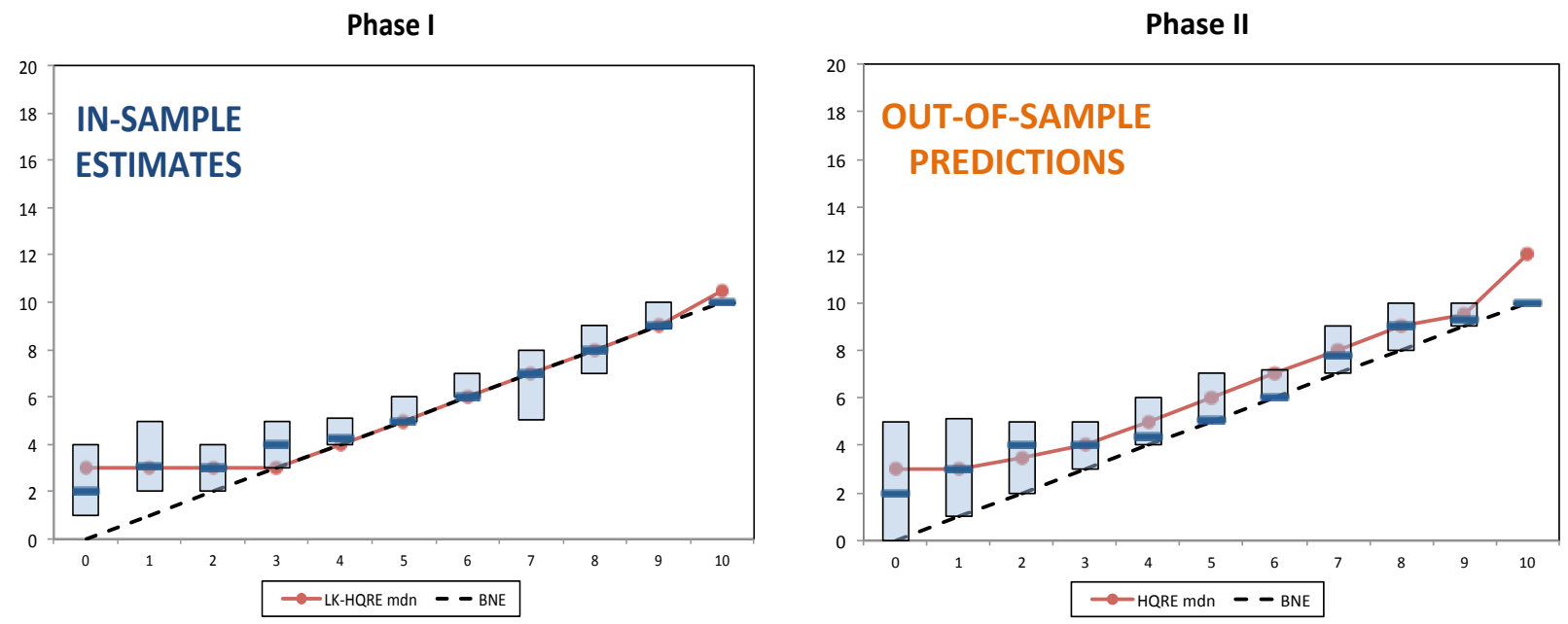

Phase I

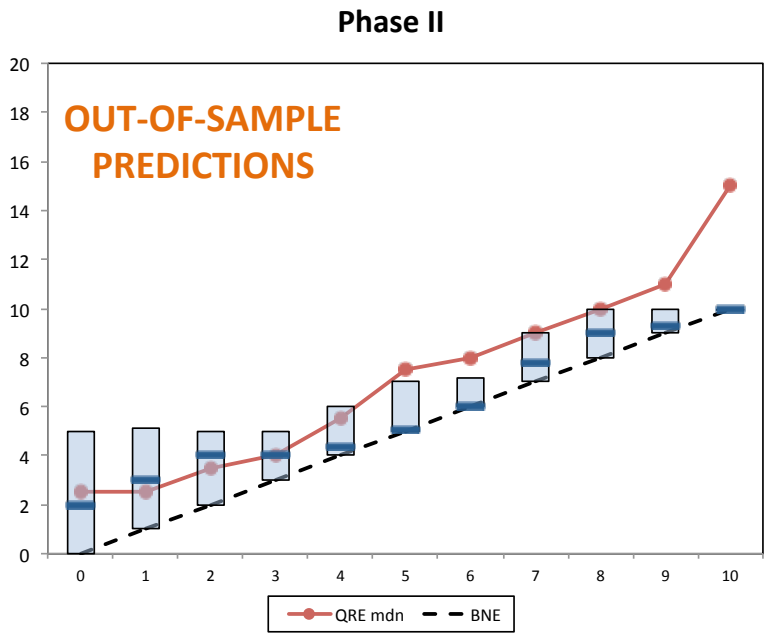

against a signal-bidder. The Phase II predictions for these almost perfect signal bidders in Phase I (who, thus, have very high estimated $\widehat{\lambda_{i}}$ 's) will necessarily put significant density on bids above signal because overbidding is nearly optimal (and for perfect signal bidders is perfectly optimal).

A second lesson from the estimation of these models concerns the role of heterogeneity. All of these models show that skill heterogeneity $\left(\widehat{\lambda_{i}} \mathrm{~s}\right)$ is a significant factor in explaining the observed behavior in this experiment. From the model estimation, there is also evidence that the second possible source of heterogeneity-heterogeneity in beliefs as captured in LK 
models - is also a significant factor. There are two pieces of evidence that support such a claim. First, there is a large and highly significant improvement in fit between the QRE and LK-QRE models (likelihood ratio greater than 100). Neither model has skill heterogeneity, so the only difference is that LK-QRE incorporates one additional parameter for belief heterogeneity. Second, of all six models, the best fitting model incorporated both skill heterogeneity and belief heterogeneity (LK-HQRE).

There are some natural questions to be raised about comparing the fits of the various models. First, the models based on HQRE have many more degrees of freedom than the homogeneous QRE-based models and some of the models are not nested in each other, so how do we discount the fit of these models for the extra degrees of freedom? A second set of questions concerns our measure of fit, which is the log of the likelihood function evaluated at the estimated parameters for each model. How does one interpret this measure of fit? Third, how do the forecasts of these models for Phase II bidding behavior compare to best reply models? This third question is particularly relevant because Phase II bidders are not bidding against "the field", which is manifestly stochastic and identical for all bidders in Phase I. Rather they are bidding against a specific non-stochastic bidding function, which is generally different for each bidder.

\subsubsection{Formal comparison of the nested and non-nested models}

We answer the first question at both the aggregate and the individual level. We start with the aggregate level. For most of the model comparisons we are comparing nested models. For example, QRE (and the hybridized QRE models) are nested in HQRE (and the hybridized HQRE models), and the QRE/HQRE models are nested in the CE-QRE/HQRE models. Thus, for these comparisons the chi-squared statistic that is used to determine significance of the likelihood ratio tests is specifically adjusted for degrees of freedom. For the QRE/HQRE tests, there are 45 degrees of freedom. For the tests comparing the QRE(HQRE) and CEQRE(HQRE) models there is one degree of freedom $(\chi)$. However, the QRE(HQRE) models are not nested in the LK-QRE(HQRE) models, because there is no value of $\tau$ that corresponds to the constrained (i.e., QRE or HQRE) model; similarly, the CE-QRE(HQRE) and LKQRE(HQRE) models are not nested in either direction. There are a variety of ways to adjust for degrees of freedom in order to properly compare the fits of non-nested models. We consider three such criteria, the Vuong's closeness test introduced by Vuong (1989), the Akaike Information Criterion (Akaike IC) introduced by Akaike (1974) and the Bayesian 
Information Criterion (Bayesian IC) introduced by Schwarz (1978), which penalize models according to the number of free parameters in different ways. ${ }^{17}$ The Akaike IC and Bayesian IC are reported in columns three and four of Table 3, and compared with the log likelihood scores (lower scores are better for all three measures). The best fitting models under the two alternative criteria are marked with an asterisk. Notice that in both cases, the best fitting model is a level-K model. According to the results of Vuong's closeness tests, the likelihood of the LK-HQRE is significantly higher than the likelihood of the HQRE model but is not significantly higher than the likelihood of the CE-HQRE model. ${ }^{18}$

Table 3: Model Comparisons Using Alternative Criteria

\begin{tabular}{l|cccc}
\hline \hline Model & $-\operatorname{lnL}$ & Akaike IC & Bayesian IC & $\phi$ \\
\hline QRE & 1447.8 & 2897.6 & 2899.4 & 0.3971 \\
HQRE & 1317.6 & 2727.2 & 2811.3 & 0.5443 \\
CE-QRE & 1440.4 & 2884.8 & 2888.5 & 0.4054 \\
CE-HQRE & 1289.7 & 2673.4 & 2759.3 & 0.5758 \\
LK-QRE & 1346.3 & 2696.6 & $2700.3^{*}$ & 0.5118 \\
LK-HQRE & $1280.9^{*}$ & $2655.8^{*}$ & 2741.7 & $0.5858^{*}$ \\
\hline
\end{tabular}

In order to compare the likelihoods of the different models at the individual level, we use likelihood ratio tests to compare HQRE with CE-HQRE and Vuong's tests to compare HQRE with LK-HQRE and CE-HQRE with LK-HQRE. We classify a subject as being best explained by a model if this model's log-likelihood is significantly higher than the log-likelihoods from both other models according to the results of these tests. Using this method, we classify 2 subjects as LK-HQRE types, 8 subjects as CE-HQRE types, and 5 subjects as HQRE types. This leaves $31 / 46$ subjects unclassified. ${ }^{19}$ The fact that most subjects are unclassified indicates that, for most subjects, all three models fit their individual bidding behavior in similar ways.

\footnotetext{
${ }^{17}$ The Vuong closeness test is a likelihood-ratio-based test for model selection using the Kullback-Leibler information criterion. See Vuong (1989) for the details. The Akaike IC is $2 k-2 L L$ and the Bayesian IC is $-2 L L+k \log (n)$ where $L L$ is the $\log$ of the likelihood function evaluated at the estimated parameters, $k$ is the number of degrees of freedom and $n$ is the size of the sample.

${ }^{18}$ The z-statistic of the Vuong's closeness test for comparing LK-HQRE with HQRE is 3.19; the z-statistics for comparing LK-HQRE with CE-HQRE is 0.73 .

${ }^{19}$ We use a $95 \%$ confidence interval to assign subjects to models. If we used a $99 \%$ confidence interval, then we would classify 2 subjects as LK-HQRE types, 5 subjects as CE-HQRE types, and 1 subject as HQRE type, leaving $38 / 46$ subjects unclassified.
} 


\subsubsection{A normalized measure of relative fit: $\phi$}

To answer the second question, we report a measure of relative fit based on the likelihood function, analogous to a Pseudo- $R^{2}$. To do so, we define an ideal "clairvoyant" model which is given by the observed conditional-on-signal bid frequencies. This is an ideal model in the sense that it predicts exactly the observed data frequencies. Call this model $M^{*}$, and the $\log$ likelihood of our data under this ideal model is denoted $\ln M^{*}$. At the other end of the spectrum is the null model that assumes behavior is totally random. Call this model Random and denote its log likelihood as $\ln$ Random. Then we define our likelihood-based measure of fit of Model $M$ as:

$$
\phi_{M}=1-\frac{\ln M-\ln M^{*}}{\ln \operatorname{Random}-\ln M^{*}}
$$

The value of $\phi$ for each model is reported in column five of Table $3 .{ }^{20}$ Two final points are worth noting. First, the random model is equivalent to the QRE model with $\lambda=0$, or the HQRE model with $\lambda_{i}=0$ for all $i$. Thus, it is certainly possible for the QRE or HQRE model to fail to explain the data at all, using $\phi$ as the measure of fit. If the estimated value of $\lambda$ were equal to 0 , then $\phi_{Q R E}=0$. Second, for best reply (as opposed to QRE) versions of the models we estimate, the likelihood of the data is 0 (and hence $-\ln L=\infty$ ), because for each of these models we have (many) observations that have zero likelihood under those models. Hence all the perfect best reply models are rejected outright.

\subsubsection{Comparison of out of sample Phase II forecasts to best reply models}

To answer the third question in a rigorous way, we compare an approximate best reply model with the out of sample Phase II forecasts based on Phase I estimates of the HQRE model, at the individual level. We use an approximate best reply model because, as pointed out earlier, a perfect best reply model makes predictions that are too sharp and would result in zero likelihood of the Phase II behavior of most subjects, and so the comparison with HQRE out of sample forecasts would be trivial as was the case in Phase I. The approximate best reply model we use perturbs the perfect best reply, by assuming players logit best respond to their Phase I actions, but with a high value of $\lambda .{ }^{21}$ This allows us to compute, for each

\footnotetext{
${ }^{20}$ In our sample, $\ln M^{*}=-914.5$ and $\ln$ Random $=-1799.0$.

${ }^{21}$ For this analysis, we use the value $\lambda=70$. The results are virtually identical for other high values of $\lambda$, such as 50 or 100 .
} 
Figure 7: Log Likelihood Ratio of Out of sample Phase II Forecast Individual Bidding Behavior to Perturbed Best Reply Model

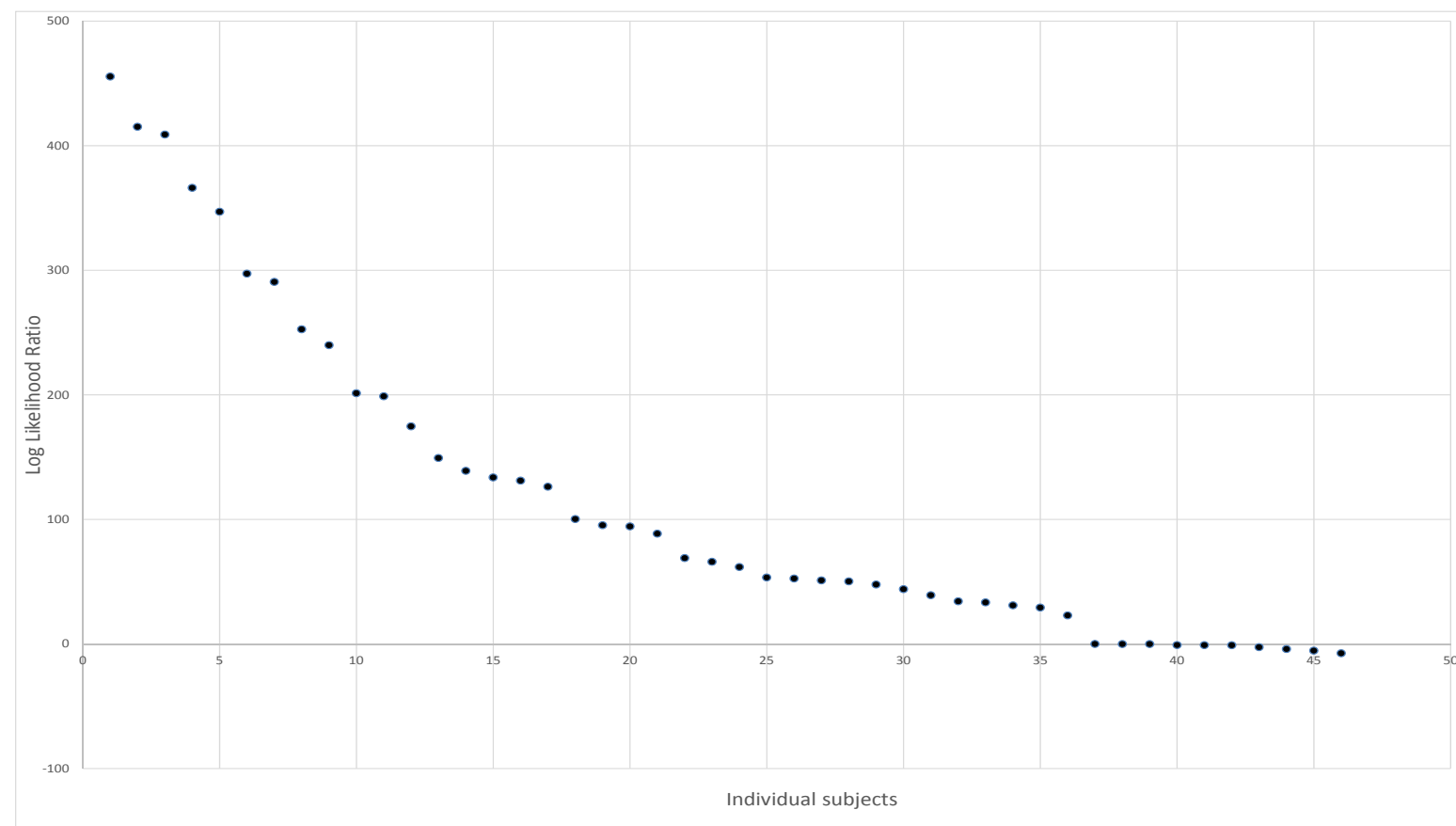

individual in the data set, the likelihood of that individual's Phase II bids under both models. This allows a direct comparison of the fit of the out of sample forecast model to the perturbed best-reply model, at the individual level, evaluated as the log of the likelihood ratio of the two models. Positive values of this ratio correspond to the out-of-sample model fitting that individual's Phase II bidding behavior better than the perturbed best-reply model, while the opposite is true for negative values. Figure 7 graphs these values for the 46 subjects in order from highest to lowest.

For all but a handful of subjects, the out of sample fits are much better than the perturbed best reply model. Specifically, for 36 subjects, the log likelihood ratio is greater than 20 (ranging from 23 to 455), clearly rejecting the perturbed best-reply model. Of the remaining 
10 subjects, 6 have a log likelihood ratio ranging from 0 to -1 , so the two models are essentially indistinguishable. The remaining 4 subjects have log likelihood ratios between -2 and -8 . This approach allows a partial model-based classification of subjects. The first 36 subjects are not best responders in Phase II, while these last 10 subjects are best responding (or nearly so) to their own Phase I bids. Thus, for these 10 subjects, nothing is gained in predicting Phase II behavior from using out-of-sample fits based on a stochastic choice model instead of a (approximately) non-stochastic best-reply model. On the other hand, very little is lost, in the sense that the Phase II bids are also predicted reasonably accurately by the out-of-sample HQRE forecasts. ${ }^{22}$ In fact, five of these subjects were also nearly perfect best responders to "the field" in Phase I as well, all five having $\lambda_{i}$ 's greater than 30.

\section{Analysis of ILN Data}

One obvious question concerns the robustness of the Section 3 results to other games and other subject pools, and a robustness check seems especially important in this case in view of an existing dataset from the experiment published by Ivanov et al. (2010). With this in mind, we performed exactly the same analysis on the treatments in the ILN experiment that are directly comparable to ours. That is, the ILN data we analyze in this section was generated from an experiment conducted with identical auction rules, procedures, instructions, and software as the data we obtained from the experiment reported above.

First, we summarize their bidding data descriptively. Figure 7 graphs the unconstrained bids by signal for Phase I, for both datasets. The left panel represents the data analyzed in Section 3 and the right panel shows the data from ILN. All bids are shown on a log scale (out of necessity, since bids can range from $\$ 0$ to $\$ 1,000,000$ ). A striking feature of ILN data which is not seen in our data is extreme overbidding. To gauge the extent of overbidding, we add to this scatterplot two lines: a continuous line drawn at a level of bidding equal to the private signal received, and a dashed line signaling a bid equal to the upper bound of the common value (i.e. $\$ 10$ ). Overall, $27 \%$ of ILN's subjects bid $\$ 100$ or more at least once. ${ }^{23}$

\footnotetext{
${ }^{22}$ To put "reasonably" in perspective, the log likelihood values for these 10 subjects based on out-of-sample forecasts range from -21 to -36 while the corresponding range for the perturbed best reply model is -16 to -35. For the remaining 36 subjects who are not approximate best responders in Phase II, the range of log likelihood values based on out-of-sample forecasts is -28 to -63 and for the perturbed best reply model the corresponding range is -60 to -513 . A totally random model would result in a log likelihood value for a subject's Phase II bids equal to -39 .

${ }^{23}$ Overall, $7.66 \%$ of bids are $\$ 100$ or above, and $2.27 \%$ of the bids are exactly $\$ 1,000,000$. These high bids
} 
These extremely high bids were not observed in the experiment reported in Section 3. Overall bids were considerably higher in the ILN data, by any measure. Table 4 provides summary descriptive statistics comparing the two datasets.

Table 4: Summary Statistics of Bids: Comparison of ILN and CNP Data

\begin{tabular}{l|cccc}
\hline \hline & \multicolumn{2}{|c}{ CNP Data } & \multicolumn{2}{c}{ ILN Data } \\
& Phase I & Phase II & Phase I & Phase II \\
\hline Average & 6.3 & 6.2 & 36,679 & 21,599 \\
First Quartile & 4.0 & 4.0 & 5.0 & 4.0 \\
Second Quartile & 5.1 & 6.0 & 7.0 & 7.0 \\
Third Quartile & 8.0 & 9.0 & 10.0 & 10.0 \\
Standard Deviation & 10.1 & 3.8 & 184,056 & 144,244 \\
Minimum & 0 & 0 & 0 & 0 \\
Maximum & 200 & 50 & $1,000,000$ & $1,000,000$ \\
\hline Subjects & 46 & 46 & 108 & 108 \\
Decisions & 506 & 506 & 1188 & 1188 \\
\hline \hline
\end{tabular}

Table 5 and Figures 8-10 present the HQRE, CE-HQRE, and LK-HQRE estimates for Phase I and out of sample predictions for Phase II, for the ILN data. In ILN, Phase II was conducted under two different protocols. In one (Baseline), subjects were not reminded how they bid in Phase I. In the other protocol (ShowBid), subjects were reminded what bids they chose for each signal in Phase I. Our experiment uses only the ShowBid procedure to avoid concerns about subjects' memory. In the analysis of ILN data, we pool together data from these two protocols, because the results are similar in their two protocols. We show the results only for the heterogeneous lambda models, because, as in the experiment reported in Section 3, all three homogeneous models are soundly rejected. ${ }^{24}$ The left panel of each figure shows predictions for Phase I obtained by estimating the parameters of the model as described in Section 3. The right panel of each figure, instead, shows the out-of-sample predictions for Phase II obtained using the parameters estimated with the data from Phase I. We only report estimates for heterogenous models because, as for our data, homogenous models are clearly rejected.

are present in all the ILN sessions.

${ }^{24}$ The extreme overbidding - for example, many bids of $\$ 1$ Million - means that some degree of heterogeneity is required in order to obtain any sort of reasonable fit to the ILN data. In fact, the estimated value of $\lambda$ in their data for the homogeneous QRE model is exactly 0. 
Table 5: Estimated Parameters and Measures of Fit

\begin{tabular}{lcc}
\hline \hline & ILN & CNP \\
\# Individuals & 108 & 46 \\
\# Observations & 1188 & 506 \\
\hline HQRE & & \\
$\widehat{\lambda}$ Q Quartiles & $(0.12,1.30,4.51)$ & $(3.40,5.12,8.70)$ \\
-Log-likelihood & 3582.0 & 1317.6 \\
$\phi$ & 0.5609 & 0.5443 \\
\hline & & \\
CE-HQRE & & 0.2 \\
$\widehat{\chi}$ & 0.5 & $(3.05,6.55,11.81)$ \\
$\widehat{\lambda}$ Q Quartiles & $3.11,1.53,6.76)$ & 1289.7 \\
-Log-likelihood & 0.5893 & 0.5758 \\
$\phi$ & & \\
\hline & & \\
LK-HQRE (4 Types) & 2.9 & $(3.82,11.06,47.51)$ \\
$\widehat{\tau}$ & $(0.12,1.88,18.05)$ & 1280.9 \\
$\widehat{\lambda}$ Q Quartiles & $(8 \%, 24 \%, 35 \%, 33 \%)$ & $(4 \%, 17 \%, 34 \%, 45 \%)$ \\
Types Frequency & 3525.8 & 0.5858 \\
-Log-likelihood & 0.6100 & \\
$\phi$ & & \\
\hline
\end{tabular}


Figure 8: Bids as a function of signals in our data (a) and ILN (2010) (b), Phase I. All bids.

(a)

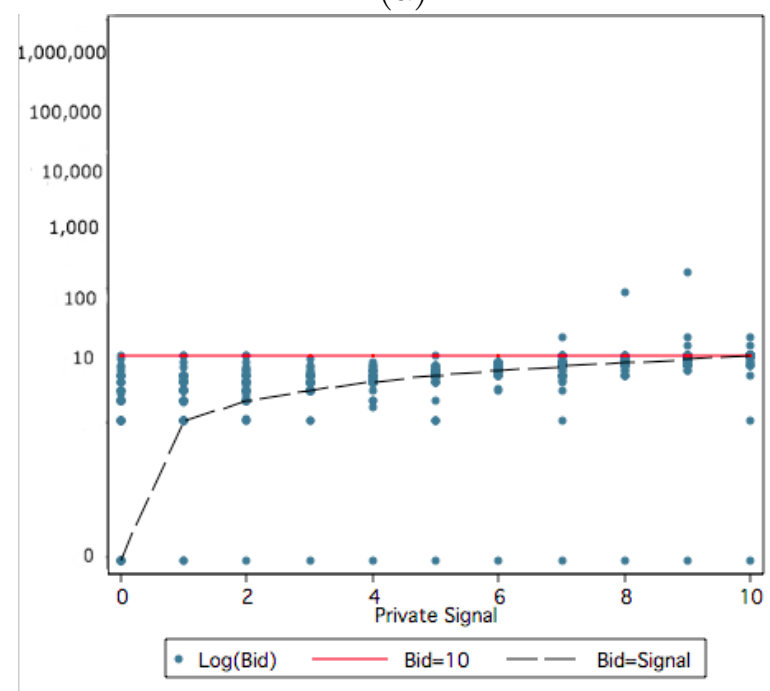

(b)

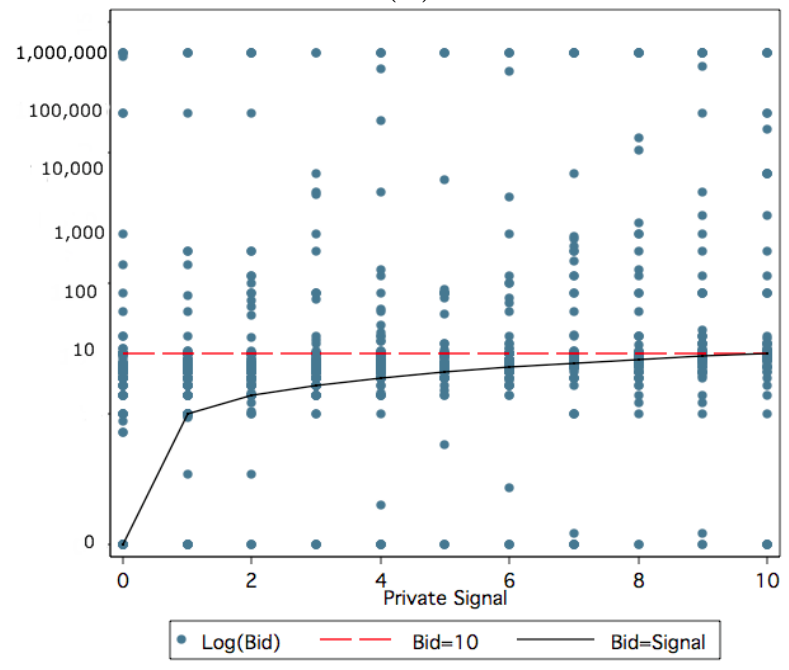

Figure 9: HQRE Predictions, ILN
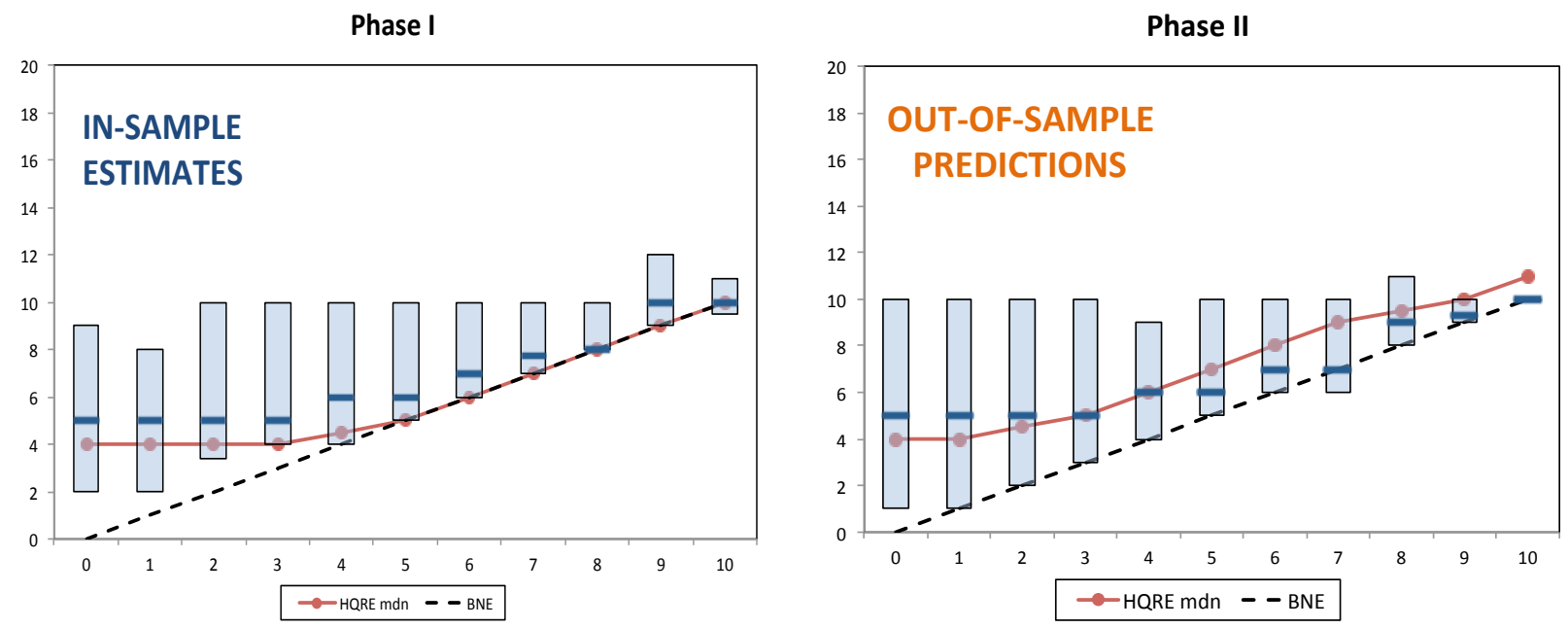

The qualitative conclusions from these estimates are similar to Section 3. As in the other dataset, all three models (HQRE, CE-HQRE, and LK-HQRE) match the overall hockeystick pattern of median bids, both in terms of fitting the Phase I data and predicting the Phase II data. Also, the best fitting model is LK-HQRE, which allows for both sources of 
Figure 10: CE-HQRE Predictions, ILN
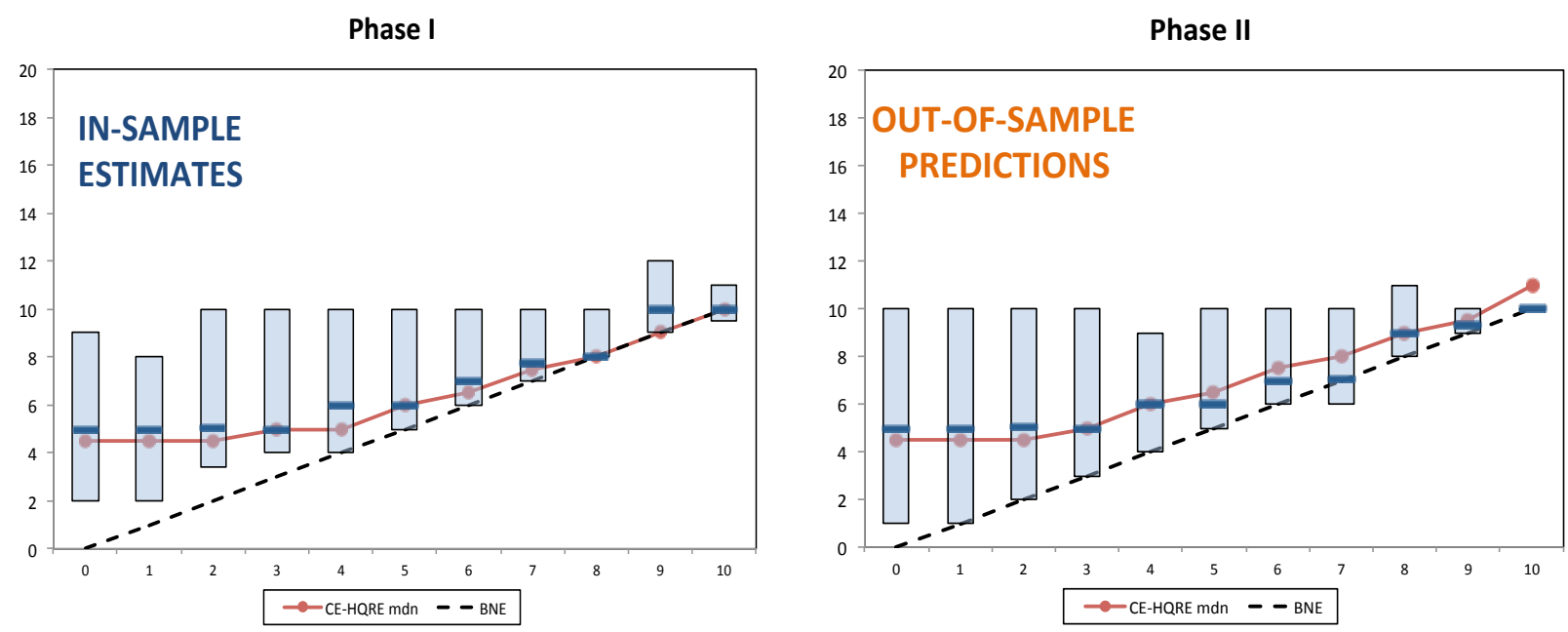

heterogeneity. The ILN data exhibit much more variance and heterogeneity compared with ours and this is borne out in the parameter estimates. Specifically, the distribution of $\lambda_{i}$ 's is shifted towards zero, the estimated distribution of level types for the LK model is lower, and the estimated degree of cursedness is higher. Nonetheless, we still find that these models combining heterogeneous quantal response and limits on strategic thinking match the basic pattern of bidding functions and fit both datasets in similar ways, even in the presence of the extreme overbidding observed in one of the datasets.

As noted earlier, CGS also critiqued ILN's methods and conclusions. CGS show that the theory in ILN does not clearly apply as those authors claim. They also show that ILN?s method of classifying bidders into level types is problematic. By expanding the definition of overbidders in a sensible and more inclusive way, CGS show that overbidders only clearly overbid in phase II for 4 of the 11 signals, so the claim that overbidders continue to persistently overbid in phase II is weak. They conclude from their re-analysis of the data that 'beliefs-based models do not necessarily imply ILN's predictions and are compatible with some observations".

An alternative to CGS is to classify bidder types in terms of their Phase II behavior in the ILN experiment, using the same method as section 3.4.3. Recall that the analysis in that section compared, at the individual level, out of sample Phase II forecasts of the HQRE model with the in-sample fitted approximate best reply model. Individuals whose Phase II 
Figure 11: LK-HQRE Predictions, ILN
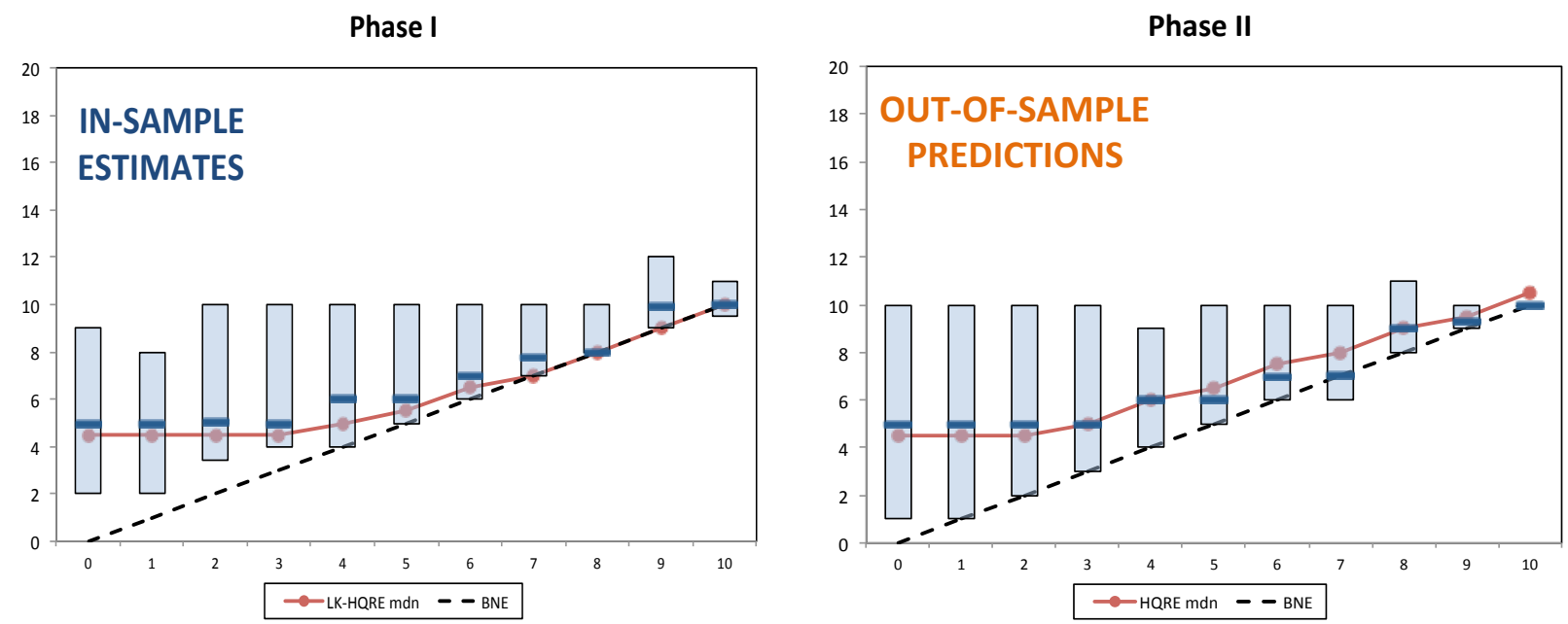

behavior is closer to the approximate best reply model than the out of sample Phase II HQRE forecasts are classified as best reply bidders. As with the CLP subject pool, we find that the out of sample forecasts are better for most subjects. Specifically, 93 out of 108 subjects are fit better with the out of sample HQRE forecast than the in-sample best reply model, and the likelihood ratio is greater than 3 for 92 of these subjects and great than 20 for 85 of them. Of the remaining 15 subjects the in-sample approximate best reply model fits better, only 7 have a likelihood ratio greater than 3. Thus, the picture is similar to what we found in the CLP data. In both subject pools about $10 \%$ of subjects are categorized as best reply or nearly best reply bidders in Phase II, based on a comparison with the out-of-sample forecasts based on the estimates of their quantal response behavior obtained in Phase I.

\section{Discussion and Conclusion}

Bidding behavior in the maximum value auction game exhibits a hockey stick-shaped pattern of overbidding. With low signals there is significant overbidding, and these overbids are relatively flat with respect to signals. With higher signals, overbidding is much less, with median bids closely tracking signals. The heterogeneous quantal response equilibrium model fits this pattern in the data, based on both within sample estimation and out-of-sample prediction. That is, that model clearly captures the hockey-stick bidding and it also generates 
approximately the right degree of variation in bids, measured by interquartile range. The fit is significantly and qualitatively improved by weakening the assumption of equilibrium beliefs (in QRE) with single parameter versions of cursed equilibrium or the level-k model with quantal response. ${ }^{25}$

It is important to emphasize that the predicted median bids in the Phase II data, which closely track the empirical median bids, are based on out-of-sample estimates obtained from the Phase I data. Moreover, in the quantal response models we explore here, the bidding behavior in Phase II is predicted to be quite similar to bidding behavior in Phase I. This is in stark contrast to models of best response behavior, where sharp differences in bidding behavior should be observed between Phase I and Phase II.

The distinction between best reply models and quantal response models is an important one because it has implications about how one evaluates the performance of belief based models based on either rational expectations (Nash equilibrium and QRE) or systematically incorrect beliefs (cursed equilibrium, ABEE, level-k, and cognitive hierarchy). If one insists on perfect best reply behavior, then all of the various belief-based models fail badly to explain the data from maximum value auction experiments. In contrast, if one allows for stochastic choice in the form of quantal responses, then a careful analysis of the data leads to the opposite conclusion, for a range of different assumptions about the structure of bidder beliefs.

We conclude with three general remarks that broadly relate to the findings of this paper. First, the maximum-value auction and associated treatments are not that useful for comparing different theories with equilibrium or non-equilibrium beliefs, under best response. All competing theories are soundly rejected by the Phase I behavior alone, in both the ILN data and the data reported here. However, in contrast, the game is actually very useful for comparing the best response and quantal response approaches. Best response models cannot explain the distribution of bids and lack of treatment effects, while quantal response models can and do. Furthermore, the quantal response approach also allows one to compare belief based models that assume rational expectations with models of non-equilibrium beliefs, and to measure the degree to which non-equilibrium beliefs are an important behavioral factor. It turns out that non-equilibrium beliefs are a significant factor in both experiments, in spite of a rather dramatic difference in bidding behavior between the two data sets. In one (ILN), there is a huge amount of variance, frequent very high overbidding, and a great deal more

\footnotetext{
${ }^{25}$ The cognitive hierarchy model with quantal response fits the data similarly to the level-k model.
} 
heterogeneity across the bidders; in the other only 12 out of 1012 bids exceed the maximum value of 10. The quantal response approach allows one to rigorously separate out the "noise" component of behavior from the systematic behavioral factors, which is virtually impossible if one views the data through the lens of perfect best-responses.

Second, the estimation and prediction analysis, using various models that combine QRE with other behavior, indicate that two distinct forms of heterogeneity are present, and can be separately identified with structural estimation: skill heterogeneity and heterogeneity in beliefs. Both are significant factors that need to be taken account of in order to understand bidding behavior in these games.

Finally, the maximum-value auction results provide an opportunity to reflect upon how much we demand, expect, and hope that models of human behavior can do. It is certainly the case that the winner's curse in common-value strategic auctions, and similar mistakes in single-person decision analogues (Carroll, Bazerman, and Maury 1988; Charness and Levin, 2009), common value bargaining settings (Carrillo and Palfrey, 2009, 2011), and other common value games (Esponda and Vespa, 2014) clearly point to a widespread failure to correctly compute - or perhaps even comprehend - conditional expectations. However, by their very construction, models specifically constructed to model the difficulty of computing conditional expectations cannot possibly explain deviations from equilibrium in complete information games or in Bayesian games with a private values structure. ${ }^{26}$ An advantage of the QRE approach, as well as the level-k and $\mathrm{CH}$ models, is that they have bite in nearly all games, that include but are not limited to only common value environments. That said, these are still very simplistic models that invite new modeling innovations. Progress beyond the useful benchmark of Nash equilibrium has been so rapid in recent years, however, that one can be optimistic about making further progress on a more integrated, predictive general theory that can be tractably applied to the empirical analysis of all these types of games and decisions.

\section{References}

[1] Akaike, Hirotogu. 1974. "A New Look at the Statistical Model identification." IEEE Transactions on Automatic Control, 19: 716-723.

\footnotetext{
${ }^{26}$ Analogy Based Expectations Equilibrium (Jehiel 2005 and Jehiel and Koessler 2008) is somewhat more general and can have interesting implications in some games with private values.
} 
[2] Bajari, Patrick, and Ali Hortacsu. 2005. "Are Structural Estimates of Auction Models Reasonable? Evidence from Experimental Data." Journal of Political Economy, 113(4): $703-741$.

[3] Brown, Alexander, Colin F. Camerer, and Dan Lovallo. 2012. "To Review or Not To Review? Limited Strategic Thinking at the Movie Box Office." American Economic Journal: Microeconomics, (2), 1-26.

[4] Bulow, Jeremy, and Paul Klemperer. 2002. "Prices and the Winner's Curse." RAND Journal of Economics, 33(1): 1-21.

[5] Camerer, Colin, Ho, Teck Hua, and Chong, Juin-Kuan. 2004. "A Cognitive Hierarchy Model of Games." Quarterly Journal of Economics, 119(3): 861-898.

[6] Camerer, Colin, Salvatore Nunnari, and Thomas R. Palfrey. 2012. "Quantal Response and Nonequilibrium Beliefs Explain Overbidding in Maximum-Value Auctions." Caltech Social Science Working Paper No. 1349.

[7] Carrillo, Juan D., and Thomas R. Palfrey. 2009. "The Compromise Game: Two-Sided Adverse Selection in the Laboratory." American Economic Journal: Microeconomics, 1(1): 151-181.

[8] Carrillo, Juan D., and Thomas R. Palfrey. 2011. "No Trade." Games and Economic Behavior, 71(1): 66-87.

[9] Carroll, John S., Max H. Bazerman, and Robin Maury. 1988. "Negotiator Cognitions: A Descriptive Approach to Negotiators' Understanding of Their Opponents." Organizational Behavior and Human Decision Processes, 41: 352-370.

[10] Charness, Gary and Dan Levin. 2009. "The Origin of the Winner's Curse: A Laboratory Study." American Economic Journal: Microeconomics, 1(1): 207-236.

[11] Chong, Juin Kuan, Colin F. Camerer, and Teck-Hua Ho. 2005. "Cognitive Hierarchy: A Limited Thinking Theory in Games." In Rami Zwick and Amnon Rapoport (Editors), Experimental Business Research: Marketing, Accounting and Cognitive Perspectives. Boston, MA: Kluwer Academic Press. 
[12] Costa-Gomes, Miguel A., Vincent P. Crawford, and Bruno Broseta. 2001. "Cognition and Behavior in Normal-Form Games: An Experimental Study." Econometrica, 69(5): 1193-1235.

[13] Costa-Gomes, Miguel A., and Makoto Shimoji. 2015. "A Comment on 'Can Relaxation of Beliefs Rationalize the Winner's Curse?: An Experimental Study." Econometrica, 83(1): 375-383.

[14] Crawford, Vincent P., Miguel A. Costa-Gomes, and Nagore Iriberri. 2013. "Structural Models of Nonequilibrium Strategic Thinking: Theory, Evidence, and Applications." Journal of Economic Literature, 51(1): 5-62.

[15] Crawford, Vincent P., and Nagore Iriberri. 2007a. "Level-k Auctions: Can a NonEquilibrium Model of Strategic Thinking Explain the Winner's Curse and Overbidding in Private-Value Auctions?" Econometrica, 75(6): 1721-1770.

[16] Crawford, Vincent P., and Nagore Iriberri. 2007b. "Fatal Attraction: Salience, Naivete, and Sophistication in Experimental Hide-and-Seek Games." American Economic Review, 97(5): 1731-1750.

[17] Esponda, Ignacio and Emanuel Vespa. 2014. "Hypothetical Thinking and Information Extraction in the Laboratory." American Economic Journal: Microeconomics, 6(4): 180-202.

[18] Eyster, Erik, and Matthew Rabin. 2005. "Cursed Equilibrium." Econometrica, 73(5): 1623-1672.

[19] Fischbacher, Urs (2007). "z-Tree: Zurich Toolbox for Ready-made Economic Experiments." Experimental Economics, 10(2): 171-178.

[20] Ho, Teck-Hua, Colin Camerer, and Keith Weigelt. 1998. "Iterated Dominance and Iterated Best Response in Experimental 'p-Beauty Contests'." American Economic Review, 88(4): 947-969.

[21] Ivanov, Asen, Dan Levin, and Muriel Niederle. 2010. "Can Relaxation of Beliefs Rationalize the Winner's Curse?: An Experimental Study." Econometrica, 78(4): 1435-1452. 
[22] Jehiel, Philippe. 2005. "Analogy-Based Expectation Equilibrium." Journal of Economic Theory, 123(2): 81-104.

[23] Jehiel, Philippe, and Frederic Koessler. 2008. "Revisiting Games of Incomplete Information with Analogy-Based Expectations." Games and Economic Behavior, 62(2): 533557.

[24] Kagel, John H., and Dan Levin. 2002. Common Value Auctions and the Winner's Curse. Princeton, NJ: Princeton University Press.

[25] McKelvey, Richard D., and Thomas R. Palfrey. 1995. "Quantal Response Equilibria for Normal Form Games." Games and Economic Behavior, 10(1): 6-38.

[26] Rogers, Brian, Thomas R. Palfrey, and Colin Camerer. 2009. "Heterogeneous Quantal Response Equilibrium and Cognitive Hierarchies," Journal of Economic Theory, 144(4): 1440-1467.

[27] Roth, Alvin. 1995. "Bargaining Experiments." In John Kagel and Alvin Roth (Editors), The Handbook of Experimental Economics. Princeton, NJ: Princeton University Press.

[28] Rubinstein, Ariel. 2007. "Instinctive and Cognitive Reasoning: A Study of Response Times." The Economic Journal, 117(523): 1243-1259.

[29] Schwarz, Gideon. 1978. "Estimating the Dimension of a Model." Annals of Statistics, 6(2): 461-464.

[30] Selten, Reinhard. 1967. "Die Strategiemethode zur Erforschung des Eingeschrankt Rationalen. Verhaltens im Rahmen eines Oligopolexperiments. "In H. Sauermann (Editor), Beitrage zur Experimentellen Wirtschaftsforschung Tubingen: J. C. B. Mohr.

[31] Stahl, Dale O.. 1996. "Boundedly Rational Rule Learning in a Guessing Game." Games and Economic Behavior, 16: 303-330.

[32] Stahl, Dale O., and Paul R. Wilson. 1995. "On Players' Models of Other Players: Theory and Experimental Evidence." Games and Economic Behavior, 10(1): 218-254.

[33] Vuong, Quang H.. 1989. "Likelihood Ratio Tests for Model Selection and Non-Nested Hypotheses." Econometrica, 57(2): 307-333. 


\section{Appendix - Experiment Instructions}

This is an experiment in the economics of market decision-making. This experiment consists of two parts and is expected to last $90 \mathrm{~min}$. The instructions are simple, and if you follow them carefully and make good decisions, you may earn a CONSIDERABLE AMOUNT OF MONEY which will be paid to you in cash at the end of the experiment.

You will receive a $\$ 5$ show-up fee which is yours to keep. In addition, you will receive 10 experimental currency units (ECU) starting cash balances for the experiment. You will also have the opportunity to earn ECU in each of the two parts of the experiment. ECU will be converted into dollars at a rate of $\$ 0.5$ per ECU (i.e., 2 ECU are worth $\$ 1$ ). Your total dollar earnings will equal:

$\$ 5$ show-up fee $+0.5 \times(10$ ECU starting cash balance + ECU earned in part I and part II $)$

Note that your earnings in part I and part II could be negative (i.e., you could incur a loss) in which case they will be subtracted from your 10 ECU starting cash balances. However, you will receive your $\$ 5$ show-up fee no matter what.

This is a serious experiment and talking, looking at others' screens, or exclaiming aloud are not allowed. Should you have any questions please raise your hand and an experimenter will come to you.

\section{PART I}

1. In part I of this experiment, we will create a series of auctions in which you will act as bidders for a fictitious item. In each auction, you will be paired randomly with another bidder. A single item will be auctioned off with the two of you as bidders. Your pairings will vary from auction to auction and will remain anonymous.

2. In each auction, you will receive a signal (call it $\mathrm{X}$ ) and the bidder that you are paired with will also receive a signal (call it $\mathrm{Y}$ ). $\mathrm{X}$ and $\mathrm{Y}$ are determined randomly and will lie between 0 and 10. Each whole number within this interval (i.e., 0, 1, 2, .., 9, 10) has an equal chance of being drawn. In addition, the value of $\mathrm{X}$ has no bearing on the value of $\mathrm{Y}$ : no matter what the value of $\mathrm{X}$, each whole number between 0 and 10 is equally likely to be the value of $\mathrm{Y}$. The value of the item that is auctioned (call it $\mathrm{V}$ ) is determined as THE LARGER of the two signals, $\mathrm{X}$ and $\mathrm{Y}$. Prior to bidding in each 
auction, you will learn $\mathrm{X}$ (but not $\mathrm{Y}$ ); the bidder you are paired with will learn Y (but not $\mathrm{X})$.

EXAMPLE 1: Suppose you learn that $\mathrm{X}=6$ and the bidder you are paired with learns that $\mathrm{Y}=4$. Then the value of the item is $\mathrm{V}=6 \mathrm{ECU}$.

EXAMPLE 2: Suppose you learn that $\mathrm{X}=1$ and the bidder you are paired with learns that $\mathrm{Y}=9$. Then the value of the item is $\mathrm{V}=9 \mathrm{ECU}$.

3. Market organization:

In each auction you will submit a bid for the item. The high bidder gets the item and makes a profit equal to the difference between the value of the item and the second highest bid. That is, for the high bidder, PROFITS $=\mathrm{V}-($ SECOND HIGHEST BID). If the difference is negative, it represents a loss. If you do not make the high bid, you will earn zero profits. In this case, you neither gain nor lose money from bidding on the item.

4. Your earnings for part I of the experiment will equal the sum of the profits you made in each auction in part I. (Because your profits in any auction could be negative, your earnings for part I could also be negative.)

5. Even though the computer will keep track of your earnings in each auction, you will not be given any feedback about the outcome of the individual auctions during the experiment.

6. No one may bid less than 0.00 ECU for the item, and bids must be rounded to two digits after the decimal point. You will have 1 minute to place your bid in each auction. In case of a tie for the high bid, the winner is chosen randomly (50-50 chance). The price the winner pays will be the second highest bid (which is the same as the high bid in case of a tie).

Let us summarize the main points:

1. High bidder gets the item and earns: V - SECOND HIGHEST BID.

2. The value of the item $\mathrm{V}$ equals THE LARGER of two signals, $\mathrm{X}$ and Y. You learn X; the bidder you are paired with learns $Y$. The signals are randomly and independently drawn from $\{0,1,2, \ldots, 9,10\}$. 
3. Your earnings for part I equal the sum of the profits you made in each auction in part I.

Any questions?

\section{PRACTICE QUIZ}

Suppose that Chris and Pat are paired for a given auction. Suppose that Chris has signal $\mathrm{X}=7$ and Pat has signal $\mathrm{Y}=4$. Suppose that Chris bids some number $B_{C h r i s}$ and Pat bids some number $B_{\text {Pat }}$. In addition, suppose for now that Chris' bid is higher than Pat's bid (i.e., $B_{\text {Chris }}>B_{\text {Pat }}$ ). Then:

1. The item is obtained by (a) Chris (b) Pat

2. The value of the item is $\mathrm{V}=$

3. The second highest bid is (a) $B_{\text {Chris }}$ (b) $B_{\text {Pat }}$

4. Chris' profits are (a) $7-B_{\text {Chris }}$ (b) $4-B_{\text {Chris }}$ (c) $7-B_{\text {Pat }}$ (d) $4-B_{\text {Pat }}$ (e) 0

5. Pat's profits are (a) $7-B_{\text {Chris }}$ (b) $4-B_{\text {Chris }}$ (c) $7-B_{\text {Pat }}$ (d) $4-B_{\text {Pat }}$ (e) 0 . Now suppose that Pat's bid is higher than Chris' bid (i.e., $B_{\text {Pat }}>B_{\text {Chris }}$ ). Then:

6. The item is obtained by (a) Chris (b) Pat

7. The value of the item is $\mathrm{V}=$

8. The second highest bid is (a) $B_{\text {Chris }}$ (b) $B_{\text {Pat }}$

9. Chris' profits are (a) $7-B_{\text {Chris }}$ (b) $4-B_{\text {Chris }}$ (c) $7-B_{\text {Pat }}$ (d) $4-B_{\text {Pat }}$ (e) 0

10. Pat's profits are (a) $7-B_{\text {Chris }}$ (b) $4-B_{\text {Chris }}$ (c) $7-B_{\text {Pat }}$ (d) $4-B_{\text {Pat }}$ (e) 0 .

PART II (Instructions read after Part I is completed)

1. We will again create a series of auctions in which a fictitious item is sold. Just like in part I, the value of the item $(\mathrm{V})$ in each auction is determined as THE LARGER of two signals, $\mathrm{X}$ and $\mathrm{Y}$. X and Y are determined randomly and will lie between 0 and 10. Each whole number within this interval (i.e., $0,1,2, \ldots, 9,10)$ has an equal chance of 
being drawn. In addition, the value of $\mathrm{X}$ has no bearing on the value of $\mathrm{Y}$ : no matter what the value of $\mathrm{X}$, each whole number between 0 and 10 is equally likely to be the value of $Y$.

2. In this part of the experiment, instead of bidding for the item against another person, you will be bidding for the item against the computer. The computer will bid by mimicking your bidding behavior from part I of the experiment (as explained below). Prior to bidding in each auction, you will observe one of the two signals $(\mathrm{X})$. The computer "observes" the other signal $(\mathrm{Y})$. Then it checks how you bid in part I when you observed that same signal and it makes the same bid.

EXAMPLE 3: Suppose that the computer observes $\mathrm{Y}=4$. Then the computer checks your bid in part I when you observed signal equal to 4 and it makes the same bid.

3. Market organization:

In each auction, you and the computer will each submit a bid for the item. If you are the high bidder you get the item and make a profit equal to the difference between the value of the item and the second highest bid. That is, if you are the high bidder, you earn: PROFITS $=\mathrm{V}-($ SECOND HIGHEST BID $)$. If the difference is negative, it represents a loss. If you do not make the high bid, you will earn zero profits. In this case, you neither gain nor lose money from bidding on the item.

4. Your earnings for part II of the experiment will equal the sum of the profits you made in each auction in part II. (Because your profits in any auction could be negative, your earnings for part II could also be negative.)

5. Even though the computer will keep track of your earnings in each auction, you will not be given any feedback about the outcome of the individual auctions during the experiment.

6. No one may bid less than 0.00 ECU for the item, and bids must be rounded to two digits after the decimal point. You will have 1 minute to place your bid in each auction. In case you tie with the computer for the high bid, you win the item with $50 \%$ chance. If you win the item, you pay a price equal to the second highest bid (which is the same as the high bid in case of a tie). 
We can summarize by saying that the rules for part II are similar to those for part I. The difference is that now you are bidding not against another participant but against the computer which mimics your bidding behavior from part I.

Any questions? 